\title{
Climate on the equilibrium line altitudes of glaciers: theoretical background behind Ahlmann's P/T diagram
}

\author{
ATSUMU OHMURA, MAXI BOETTCHER \\ Institute for Atmospheric and Climate Science (IAC), Swiss Federal Institute of Technology (E.T.H.), Zurich, Switzerland \\ Correspondence: Atsumu Ohmura <ohmura@env.ethz.ch>
}

\begin{abstract}
The climatic condition that prevails at a glacier equilibrium line altitude (ELA) is often parameterized in terms of summer air temperature $(I)$ and annual precipitation $(P)$. This simple parameterization was initially proposed by Hans W:son Ahlmann. The physical background of the relationship between $\boldsymbol{P}$ and $T$ on the equilibrium line, however, has been left unexplained since Ahlmann first questioned the mathematical form of the relationship. This relationship can be explained when the thermal and hydrological processes of the ELA formation are investigated. The present authors studied the energy exchange processes that prevail on the ELA during the melt season. The inclusion of solar radiation brings Ahlmann's hypothesis closer to energy balance, and improves his $P / T$ diagram. By comparing the observed fluxes from the polar through the mid-latitude to the equatorial glaciers, it was found that these glaciers in different climatic regions share important similarities at the ELA. Further, it was found that the classic $P / T$ curve originally proposed by Ahlmann in the early 20th century is a concise expression of the conservation principle of energy and mass at the ELA of glaciers, and takes the form of a polynomial of the fourth order.
\end{abstract}

KEYWORDS: accumulation, glacier mass balance, ice and climate, ice/atmosphere interactions, surface mass budget

\section{INTRODUCTION}

The line or a zone on a glacier where the surface annual mass balance converges to zero is defined as the equilibrium line, and its altitude above the mean sea level is the equilibrium line altitude (ELA) (Cogley and others, 2011). Traditionally, glaciologists tended to consider the ELA in terms of the mean ELA averaged over a certain period, which was long enough to be climatic.

The ELA by definition divides the glacier surface into an accumulation and ablation area. This implies that the ELA represents the lowest boundary of the climatic glacierization, the existence of the ablation area being the consequence of the ice rheology. For the existence of glaciers, it is necessary that the ELA lies below topographic and glacier surfaces. Consequently, the upper limits of the lateral and medial moraines lie very close to the equilibrium line, a feature which is used in quaternary geology to estimate the ELA of the past (Hantke, 1978; Ballantyne, 1989). If the ELA is lifted above the highest bedrock or ice surface topography as a result of climate change, the glacier is doomed to disappear.

The ELA is also a useful concept as it is closely related to the annual mass balance. Where information of annual mass balance is lacking, the ELA provides an alternative way of estimating the annual mass balance (Liestøl, 1967; Paterson, 1969; Koerner, 1970; Schytt, 1981; Kulkarni, 1992; Seversky, 1997; Nesje and Dahl, 2000). This aspect also offers a method to estimate the change in mass balance as a result of climate change (Bradley, 1975; Kuhn, 1980, 1989; Ambach, 1989, 1993). Further, the altitudinal distributions of the annual mass balance, and especially of the summer balance, show the largest variations at or near the ELA (Ohmura and others, 1992). These features can be exploited to identify the ideal location for monitoring the atmosphere on the glacier in order to predict the meltwater discharge for agricultural irrigation and hydroelectric power generation. Therefore, the parameterization of the ELA is of primary importance not only for the theory of the glacier/climate relationship but also for applications such as water economy.

The glacier/climate relationship on the ELA was first expressed as a relationship between precipitation $(P)$ and temperature ( $T$ ) by Ahlmann (1924), in the form of what was later called the Ahlmann's P/T diagram. The diagram indicated a non-linear relationship between precipitation and temperature, which Ahlmann himself questioned. He considered two possible forms of the relationship, a second-order polynomial and exponential functions. Later workers also noticed the non-linearity of the relationship, and came up with an exponential curve (Ballantyne, 1989) and various polynomials (Kotlyakov and Krenke, 1982; Ohmura and others, 1992; Krenke and Khodakov, 1997). Andrews (1975) fitted the curve with three different straight line segments. More recently, Nesje and Dahl (2000) again used exponential curves. Authors used their intuition for selecting a function and fitting regression lines based on statistical means. The physical processes on ELAs, hence the physically based mathematical form of the function, have been left unidentified up to the present.

The objective of the present paper is (1) to examine the $\mathrm{P} / \mathrm{T}$ relationship for the glaciers under diverse physical conditions, (2) to investigate the climatic conditions that determine the mean ELA with more abundant data of higher quality and (3) to present the main physical processes on ELAs in order to identify a mathematical form of the $\mathrm{P} / \mathrm{T}$ diagram based on the theory of mass and energy conservation. 


\section{THE DEVELOPMENT OF ELA PARAMETERIZATION}

\subsection{Choice of parameters}

The meteorological elements used in the present work are air temperature, precipitation and global solar radiation, three quantities that represent three major terms in the energybalance equation for the glacier surface, as will be detailed later. Prior to the knowledge of the energy balance, the importance of temperature and precipitation was already known in the second half of the 19th century (Forbes, 1853; Hann, 1883). Forel and others (1909) and Maurer (1914) thought solar radiation to be a secondary but non-negligible heat source. It was however Ahlmann (1924) who characterized the ELA numerically by using temperature and precipitation, but abandoned solar radiation as unconvincing. The present paper demonstrates that all three elements are necessary.

For the air temperature, the free atmospheric temperature is preferred to the local temperature on the glacier. Lang (1968) reported a higher correlation between the melt and the air temperature measured outside the glacier. Using an extra-glacier temperature was also chosen later by Vincent (2002), Mernild and others (2013) and Pelto and others (2013). The present authors credit this tendency to the difficulty of defining the optimal temperature on the glacier owing to the strong temperature gradient near the glacier surface with respect to height and the horizontal expanse. Considering the temperature distribution over the glacier as the problem of turbulent heat diffusion, under the lower boundary condition of the melting temperature of the ice surface, which is constant, and the upper and lateral boundary conditions of external air, the temperature measured outside the direct glacier influence can be considered as the boundary conditions. Since the surface temperature is constant, the external air temperature becomes a sole factor to determine the solution. The air temperature between the glacier surface and the upper and lateral boundaries of the considered air volume becomes a solution with a strong vertical and horizontal gradient, which is impractical to characterize the glacier climate. In fact, if one approaches the glacier surface close to the roughness length, the air temperature is expected to become the melting point, and loses information on the energy fluxes. The external boundary condition is a more stable information-carrying parameter. The adoption of the free atmospheric temperature has another advantage compared with the near-glacier surface temperature. The free atmospheric temperature is much more easily and widely available, and also saves the cumbersome and uncertain task of downscaling (Ohmura and others, 1992), as is also shown by Rasmussen and Wenger (2009). The mean temperature of the three summer months of June, July and August (December, January and February for the Southern Hemisphere) is used as most of the annual ablation for the majority of glaciers at the ELA occurs during these three months.

For precipitation, the annual total precipitation represents annual accumulation better than the winter precipitation alone as precipitation in summer is often solid at high altitudes (Ahlmann, 1922; Müller-Lemans and others, 1993, 1997; Seversky, 1997; Braithwaite, 2008; Barrueto, 2009). Further, summer precipitation suppresses the melt through the higher albedo, larger cloud amount and lower air temperature (Ohmura and others, 1992). There are also regions where the main accumulation happens in summer, as in the interior of the Asian continent and the tropical Andes (Ohmura and others, 1990; Kang and others, 1996). These facts support the choice of annual precipitation rather than the winter accumulation or precipitation alone.

The present work chose annual precipitation as in the original works by Ahlmann (1922, 1924). However, the authors consider the possibility of a partial loss of the summer precipitation from the accumulation when it falls as liquid precipitation. To investigate the influence of the liquid precipitation on annual accumulation, the liquid precipitation at high-altitude observatories was evaluated, where the type and amount of the precipitation were observed by observers. The measured liquid precipitation at high-altitude stations is presented in Table 1, along with other information that helps to interpret the effect of the liquid precipitation on mass balance. When the observatory is located more than $200 \mathrm{~m}$ below the mean ELA, a temperature correction with a lapse rate of $0.65 \mathrm{~K} / 100 \mathrm{~m}$ (Fliri, 1975; Schüepp and others, 1978; Auer and others, 2001) and liquid portion correction of $8 \% / \mathrm{K}$ (Ohmura and others, 1999) was applied. The present survey shows that on an average, 9\% of the annual total precipitation fell in liquid form at ELA during the summer. Although a part of the liquid precipitation might remain in the snow and firn layers as superimposed ice, this amount should be born in mind as a potential loss that would be unaccounted for as a contribution to the accumulation.

Another possibility for estimating the annual precipitation at the ELA is a unique method proposed by Braithwaite and others (2006) and Braithwaite (2008). Braithwaite's method consists of estimating the annual melt based on the positive degree-days at ELA, which by definition must be the same as the annual accumulation. The uncertainty of this method is the variability of the degree-day coefficient. Once this problem is clarified, the present authors consider Braithwaite's method as a powerful method for estimating annual precipitation for high altitudes.

When Ahlmann (1924) presented the precipitation (ordinate) and temperature (abscissa) diagram (1924, $P / T$ diagram in Fig. 5, P. 264), the relationship showed a certain scatter around the best-fit curve. Later works, for example, Loewe (1971) and Ohmura and others (1992), had an even larger scatter, mainly because more glaciers from much larger regions with diverse climatic conditions were considered. The authors realized that the adoption of a third variable besides $P$ and $T$ would reconcile the scatter in a systematic manner. This third variable was found to be solar global radiation (Forel and others, 1909; Maurer, 1914; Loewe, 1971; Ohmura and others, 2007).

\subsection{Source for glaciological data}

A single most comprehensive source of the surface mass balance and ELA is the ten volumes of Fluctuations of Glaciers, started by Kasser (1967), succeeded by Müller (1977), Haeberli and others (1985) and continued by Zemp and others $(2011,2012)$ (WGMS, 2012; World Glacier Monitoring Service). This series contains data covering the 51 years from 1959 to 2010 . However, the series of publications was regularly updated with data older than 1959 that became newly available. The oldest mass balance and ELA published in this series is that of the Taku Glaciers starting in 1945. The information related to this series is available at http://wgms.ch/glacierapp/. Supplementary data are 
obtained from the series of 12 issues of Glacier Mass-Balance Bulletin started by Haeberli and Herren (1991) and continued to WGMS (2013) covering the period of 24 years from 1988 to 2011. The data for more recent years after Vol. 12 are available in WGMS (2015) under the new series title of Global Glacier Change Bulletin, and also directly from the WGMS. The current data at WGMS are available at http:// wgms.ch/ggcb/. However, the data available from the website are by no means accurate and complete, and require corrections and amendments for which the glaciology community can contribute. There are a few other sources at the national level, for example, the results of the mass-balance survey published in Glasiologiske Undersökelser i Norge (Glaciological investigations in Norway) for glaciers in Norway, starting with Østrem and Liestøl (1964) and continued to Kjøllmoen (2011). The longest series of observation contained in this series is from Storbreen starting in 1949. Many research groups publish the results of the observations carried out during their projects, which often contain detailed information on mass balance and ELA (e.g. Cogley and others, 1995, for the White Glacier and Baby Glacier; Escher-Vetter and others, 2012, for Vernagtferner). For some glaciers, the pre-publication data of the mass balance and ELA were made available, for example, for Hofsjökull by T. Thorsteinsson of Icelandic Meteorological Service and for Langjökull by F. Pálsson of the University of Iceland. The oldest and the longest massbalance/ELA observation (starting in 1914/15) has been carried out on Claridenfirn in the Glarner Alps by four generations of meteorologists at the Swiss Federal Office of Meteorology and Climatology where the data are available.

The mass-balance data are often re-evaluated after the publications. The authors encountered up to four different massbalance evaluations for the same glacier. This sort of situation is not uncommon working with the mass-balance data. Usually, there is a legitimate reason for the re-evaluation, which makes usually the later re-evaluations better in quality. One of the important re-evaluations of the annual data is made by adjusting the annual data against geodetic survey. Some examples are the White Glacier on Axel Heiberg Island (Cogley and others, 1995; Thomson and others, 2017), Storglaciären in Sweden (Holmlund and others, 2005) and ten Norwegian glaciers (Andreassen and others, 2016, http:// glacier.nve.no/viewer/Cl/en/). Whenever necessary, the authors consulted the scientists involved in the original observations, evaluations and the subsequent re-evaluations for clarifying the reasons for the differences. The mean ELA and winter balance $\left(B_{\mathrm{w}}\right)$, which are averaged over the available period of the data, are presented in Table 2 .

\subsection{Source for meteorological data}

The free atmospheric temperature at the ELA was extracted from ERA-Interim Reanalysis (Dee and others, 2011). The temperature in the Antarctic coastal zones in the ERAInterim is, however, greatly underestimated due to the inaccuracy of the model topography. For this zone, the climatological maps of the Free University of Berlin were used (Scherhag, 1969). There are at present no re-analyses that can reproduce precipitation accurately. The observed annual total precipitation near ELAs is available in limited cases from the national meteorological networks (e.g. glaciers in the Hohe Tauern in Austria, and in the eastern Swiss Alps). The observations made at high altitudes are 
also available in HISTALP (Historical Instrumental climatological Surface Time series of the greater Alpine region, Auer and others, 2007) and APGD (Alpine Precipitation Grid Dataset, Isotta and others, 2014), but direct observations near the ELA are rare, simply because there are few observatories and projects at such locations lasting long enough to provide climatic data. In the present paper, the measurements made by automatic stations are excluded, as the accuracy of the automatic stations at high altitudes is highly uncertain (Auer, 1992; Müller-Lemans and others, 1993; 1997; Barrueto, 2009). The best alternative for the annual precipitation at ELA, which is adopted in the present work, is to sum the winter accumulation $\left(B_{\mathrm{w}}\right)$ at the equilibrium line and the precipitation measured during summer at an altitude close to ELA (Ohmura and others, 1992; Braithwaite, 2008). Although the observations of annual precipitation at high altitudes are rare, there are a few observations made in summer, as many glaciological summer campaigns measure precipitation at high altitudes (e.g. Ohmura and Müller, 1978; Weber, 1997; WGMS, 2015). Only when the summer precipitation was not available, but winter accumulation was thought to be reliable, the ERA-Interim monthly precipitation from May to September (October to March in the Southern Hemisphere) was used in place of the locally measured summer precipitation. The present study, however, showed that the ERA-Interim underestimated precipitation by $17-40 \%$ for the glacierized regions, as indicated in columns 6 and 9 in Table 2. ERA-Interim evaluation presented by de Leeuw and others (2015) found a mean underestimation of $22 \%$ based on a comprehensive study for Great Britain. Therefore, when the ERA-Interim summer precipitation was used, it was increased by $22 \%$.

As stated earlier in Section 2.1, the present work introduces solar global radiation as the third variable. This decision is based on the ten energy-balance measurements summarized in Section 3.2, which shows that solar radiation is the second largest heat source for the melt on all glaciers. The largest collection of solar global radiation is available in the Global Energy Balance Archive (GEBA, a part of Baseline Surface Radiation Network/World Climate Research Program and Global Climate Observing System) at E.T.H. Zurich. Presently, GEBA harbors monthly global solar radiation for 1700 sites (Wild and others, 2017). The observation sites are dense enough for the present purpose for the Alps, Scandinavia and polar regions. For the radiation with more than $1000 \mathrm{~m}$ altitude difference, a correction was applied with the assumption of the vertical radiation gradient of $+1 \mathrm{~W} \mathrm{~m} \mathrm{~m}^{-2} / 100 \mathrm{~m}$ (Marty, 2000). The radiation from the areas with sparse observational networks, such as the high mountain regions of Asia, Latin America and Africa, global solar radiation was obtained from the GCM simulation with ECHAM4 T106. The shortwave irradiance simulated by ECHAM4 T106 was found to come closest to the observed values among all GCMs that participated in the Atmospheric Model Inter-comparison Program, AMIP II (Wild and others, 1998). Radiation data sources are presented for each glacier in Table 2.

\section{NEW P/T DIAGRAM AND ENERGY BALANCE ON ELA DURING THE MELT SEASON}

\subsection{New $P / T$ diagram based on selected glaciers}

Out of more than 130 glaciers that have medium- to longterm observations of winter and summer balances and ELA,
104 glaciers with proximity to manned meteorological observations were selected (Table 2). The glaciers were also selected to represent major glacierized regions of the world. The geographical distribution of the 104 glaciers and their mean ELAs are illustrated in Figure 1. The new $\mathrm{P} / \mathrm{T}$ diagram was constructed based on the data from 104 glaciers presented in Table 2, and illustrated in Figure 2. The correlation coefficient is 0.73 , while the coefficient of determination is 0.63 . Table 2 also contains all glaciological and meteorological data sources discussed in Section 2. The least-square linear and quadratic regression lines of all 104 glaciers are also presented in Figure 2. The numerical nature of the regression lines will be discussed later in Section 4. It was found that relatively large scatter of the diagram can be systematically grouped into narrow ranges according to solar global radiation as the third variable. The four curves in Figure 3 in Section 3.2 represent the best-fit linear regressions for the four groups, each spanning a range of $25 \mathrm{~W} \mathrm{~m}^{-2}$. In the present study, the largest summer global radiation found was $305 \mathrm{~W} \mathrm{~m}^{-2}$ in the Pamir, while the smallest was $165 \mathrm{~W} \mathrm{~m}^{-2}$ in Svalbard. These relationships will be interpreted in light of the energy balance of the melt period in the next section.

\subsection{Energy balance at ELA during the melt period}

There are nine glaciers for which the full melt season energybalance measurements were carried out in the equilibrium line areas. Table 3 summarizes the mean fluxes of the energy balance of individual glaciers. These glaciers represent a wide range of climatic conditions. They represent polar, mid-latitude and tropical glaciers. In terms of the ELAs, they range almost from the sea level to $5000 \mathrm{~m}$ a.s.l. The first four sites in Table 3 represent glaciers near the sea, while the following five sites the glaciers in continental interiors. The tenth experiment took place on a tropical high-altitude glacier. Despite the wide range of climatic conditions, these glaciers reveal strikingly similar energy-balance features during the melt period. Common to all glaciers is the dominance of longwave incoming radiation from the atmosphere. For all glaciers, this component is on average $70 \%$ of the total energy income, with extremes of maximum $86 \%$ (Meighen Ice Cap) to minimum 57\% (Blue Glacier). For all glaciers, the absorbed solar radiation takes the second place as an energy source, on an average $25 \%$ of the total energy income, with extremes of maximum 35\% (Vernagtferner) to minimum 15\% (Meighen Ice Cap). The fact that the absorbed solar radiation is the second most important energy source at ELA was earlier recognized (Ohmura, 2001) and also used for improving the melt model (Pellicciotti and others, 2005). Sensible heat flux, or turbulent enthalpy flux, is $6 \%$ of the total energy on an average, with extreme cases of $19 \%$ (Rhonegletscher) and $-1 \%$ (Meighen Ice Cap). The most important feature of the energy balance of ELA is the fact that longwave and shortwave incoming radiative fluxes are by far the dominant sources and turbulent components are one order of magnitude smaller. Since the major portion of the longwave radiation originates from the lowest $1 \mathrm{~km}$ of the atmosphere (Ohmura, 2001), the air temperature becomes particularly important information for calculating the melt. The majority of the energy gained by the surface is expended for radiative emission by the surface, which is almost constant near at the blackbody emission of the ice melting point, $315.7 \mathrm{~W} \mathrm{~m}^{-2}$ 
Table 2. Equilibrium line altitude (ELA), temperature, precipitation, global solar radiation and their sources

\begin{tabular}{|c|c|c|c|c|c|c|c|c|c|c|c|c|c|c|}
\hline Glacier & Geographic & Coordinates & ELA m a.s.l. & $\begin{array}{l}\mathrm{T}_{\text {JIA }} \\
\text { ERA- } \\
\text { Interim }\end{array}$ & $\begin{array}{l}\mathrm{T}_{\text {JJA }} \\
\text { FUB. } \\
\text { Atmos. }\end{array}$ & $\begin{array}{l}\text { Ann. Precip. } \\
\text { mm, ERA- } \\
\text { Interim }\end{array}$ & $\begin{array}{l}\text { Ann. } \\
\text { Precip. } \\
\text { APGD mm }\end{array}$ & $\begin{array}{l}\text { Bw mm } \\
\text { w.e.obs. } \\
\text { at ELA }\end{array}$ & $\begin{array}{l}\text { Bw }+ \\
\text { Psummer, } \\
\text { mm }\end{array}$ & $\begin{array}{l}\text { ELA observed } \\
\text { years }\end{array}$ & $\begin{array}{l}\text { Global } \\
\text { radiation, } \\
\mathrm{W} \mathrm{m}^{-2}\end{array}$ & $\begin{array}{l}\text { Mass balance and precip. } \\
\text { data source }\end{array}$ & $\begin{array}{l}\text { Organization for } \\
\text { mass balance obs. }\end{array}$ & $\begin{array}{l}\text { Radiation } \\
\text { data source }\end{array}$ \\
\hline Canadian Arctic & latitude & longitude & & ${ }^{\circ} \mathrm{C}$ & ${ }^{\circ} \mathrm{C}$ & & & & & & & & & \\
\hline $\begin{array}{l}\text { Ward Hunt Ice Shelf/ } \\
\text { Rise }\end{array}$ & $83^{\circ} 07^{\prime} \mathrm{N}$ & $74^{\circ} 10^{\prime} \mathrm{W}$ & 0 & -0.87 & -1.0 & 195 & & 180 & 240 & 1959-84 & 207 & 1,2 & DRB & $1,2,36$ \\
\hline Gilman Glacier & $82^{\circ} 10^{\prime} \mathrm{N}$ & $71^{\circ} 15^{\prime} \mathrm{W}$ & 1250 & -1.84 & -1.1 & 116 & & 110 & 170 & 1951-61 & 240 & 3,4 & $\begin{array}{l}\text { Geogr. Branch, } \\
\text { Ottawa }\end{array}$ & 1 \\
\hline Per Ardua & $81^{\circ} 27^{\prime} \mathrm{N}$ & $76^{\circ} 35^{\prime} \mathrm{W}$ & 1350 & -2.05 & -1.5 & 106 & & 150 & 190 & 1967-68 & 242 & 5 & ETH & 1,3 \\
\hline White Glacier & $79^{\circ} 30^{\prime} \mathrm{N}$ & $90^{\circ} 58^{\prime} \mathrm{W}$ & $1066 \pm 255$ & -0.54 & -1.6 & 194 & & 180 & 320 & 1960-13 & 239 & $6,37,67$ & $\begin{array}{l}\text { McGill, ETH, Trent } \\
\text { Univ. }\end{array}$ & $1,4,5,6$ \\
\hline Baby Glacier & $79^{\circ} 26^{\prime} \mathrm{N}$ & $90^{\circ} 58^{\prime} \mathrm{W}$ & $973 \pm 117$ & -0.26 & -1.7 & 199 & & 200 & 350 & 1965-76 & 233 & 7,37 & $\begin{array}{l}\text { McGill, ETH, Trent } \\
\text { Univ. }\end{array}$ & 1,7 \\
\hline Laika Glacier & $75^{\circ} 53^{\prime} \mathrm{N}$ & $79^{\circ} 10^{\prime} \mathrm{W}$ & 355 & 1.4 & 0.5 & 305 & & 410 & 590 & 1972-79 & 270 & 8 & ETH & 1,8 \\
\hline Devon Ice Cap NW & $75^{\circ} 15^{\prime} \mathrm{N}$ & $82^{\circ} 00^{\prime} \mathrm{W}$ & $1154 \pm 248$ & -0.74 & -0.3 & 274 & & 180 & 240 & 1961-13 & 221 & 9,10 & PCSP & 1,9 \\
\hline $\begin{array}{l}\text { Barnes Ice Cap, } \\
\text { South Dome }\end{array}$ & $70^{\circ} 10^{\prime} \mathrm{N}$ & $73^{\circ} 30^{\prime} \mathrm{W}$ & $810 \pm 151$ & 1.98 & 1.9 & 355 & & 440 & 590 & 1970-84 & 202 & 1,11 & $\begin{array}{l}\text { Glaciol. Div., } \\
\text { Ottawa }\end{array}$ & 1 \\
\hline Decade Glacier & $69^{\circ} 39^{\prime} \mathrm{N}$ & $69^{\circ} 55^{\prime} \mathrm{W}$ & 1175 & 0.7 & -0.3 & 330 & & 240 & 310 & 1965-70 & 196 & 5,37 & $\begin{array}{l}\text { Glaciol. Div., } \\
\text { Ottawa }\end{array}$ & 1,33 \\
\hline \multicolumn{15}{|c|}{ Greenland } \\
\hline Greenland Ice Sheet & $76^{\circ} 30^{\prime} \mathrm{N}$ & $68^{\circ} 00^{\prime} \mathrm{W}$ & 650 & 1.6 & 0.6 & 307 & & 410 & 700 & Stratigraphic & 225 & 12,13 & CRREL & 3,36 \\
\hline Greenland Ice Sheet & $69^{\circ} 30^{\prime} \mathrm{N}$ & $48^{\circ} 20^{\prime} \mathrm{W}$ & 1200 & -0.87 & 1.1 & 497 & & 420 & 510 & 1982-89 & 212 & 14 & GGU & $3,10,11$ \\
\hline Greenland Ice Sheet & $64^{\circ} 30^{\prime} \mathrm{N}$ & $49^{\circ} 32^{\prime} \mathrm{W}$ & 1310 & 2.77 & 2.0 & 739 & & 590 & 990 & 1981-84 & 208 & 17 & GGU & 3 \\
\hline Qapiarfiup sermia & $63^{\circ} 36^{\prime} \mathrm{N}$ & $52^{\circ} 08^{\prime} \mathrm{W}$ & 790 & 4.06 & 2.9 & 941 & & 1120 & 1350 & 1981-85 & 200 & 15,16 & GGU & $3,12,33$ \\
\hline $\begin{array}{l}\text { Greenland Ice Sheet } \\
\text { Iceland }\end{array}$ & $61^{\circ} 30^{\prime} \mathrm{N}$ & $45^{\circ} 23^{\prime} \mathrm{W}$ & 1500 & -0.44 & 2.3 & 1094 & & 590 & 870 & 1979-83 & 228 & 17, ERA-Interim & GGU & 13 \\
\hline Hofsjokull N & $64^{\circ} 57^{\prime} \mathrm{N}$ & $18^{\circ} 55^{\prime} \mathrm{W}$ & $1327 \pm 81$ & 2.41 & & 1027 & & 1457 & 1740 & 1988-14 & 200 & $36,37,38,39,40,60,76$ & $\mathrm{IMO}$ & $12,33,36$ \\
\hline Hofsjokull SW & $64^{\circ} 43^{\prime} \mathrm{N}$ & $19^{\circ} 03^{\prime} \mathrm{W}$ & $1341 \pm 75$ & 2.42 & & 1154 & & 1522 & 1916 & $1991-14$ & 200 & $37,38,39,40,52$ & $\mathrm{IMO}$ & 12,36 \\
\hline Vatnajökull SE & $64^{\circ} 20^{\prime} \mathrm{N}$ & $16^{\circ} 00^{\prime} \mathrm{W}$ & $1100 \pm 123$ & 3.68 & 4.0 & 1432 & & 2000 & 2600 & Stratigraphic & 202 & 18 & $\begin{array}{l}\text { Univ. Iceland, } \\
\text { NPCR }\end{array}$ & $3,12,36$ \\
\hline \multicolumn{15}{|l|}{ Svalbard } \\
\hline Austfonna & $79^{\circ} 30^{\prime} \mathrm{N}$ & $25^{\circ} 15^{\prime} \mathrm{E}$ & 335 & -0.08 & 0.5 & 435 & & 850 & 940 & Stratigraphic & 170 & 19,77 & SPRI & 3,12 \\
\hline Austre Breggerbreen & $78^{\circ} 53^{\prime} \mathrm{N}$ & $11^{\circ} 50^{\prime} \mathrm{E}$ & $432 \pm 98$ & 0.65 & & 497 & & 650 & 800 & $1967-14$ & 165 & $\begin{array}{l}5,35,36,37,38,39,40,50, \\
51,52,53,77\end{array}$ & NPI & 12,36 \\
\hline Midtre Lovenbreen & $78^{\circ} 53^{\prime} \mathrm{N}$ & $12^{\circ} 04^{\prime} \mathrm{E}$ & $407 \pm 89$ & 0.71 & & 479 & & 693 & 843 & 1968-14 & 165 & $\begin{array}{c}5,35,36,37,38,39,40,50 \\
51,52,53,77\end{array}$ & NPI & 12,36 \\
\hline Austre Lovenbreen & $78^{\circ} 52^{\prime} \mathrm{N}$ & $12^{\circ} 09^{\prime} \mathrm{E}$ & 431 & 0.64 & & 479 & & 715 & 920 & 2008-14 & 165 & $\begin{array}{l}\text { WGMS database, ERA- } \\
\text { Interim }\end{array}$ & UFC & 12,36 \\
\hline Irenebreen & $78^{\circ} 39^{\prime} \mathrm{N}$ & $12^{\circ} 06^{\prime} \mathrm{E}$ & $473 \pm 92$ & 0.7 & & 507 & & 522 & 772 & $2002-13$ & 165 & $\begin{array}{l}\text { WGMS database, ERA- } \\
\text { Interim }\end{array}$ & $\begin{array}{l}\text { Inst. Geography, } \\
\text { Torun }\end{array}$ & 12,36 \\
\hline Waldemarbreen & $78^{\circ} 40^{\prime} \mathrm{N}$ & $12^{\circ} 00^{\prime} \mathrm{E}$ & $427 \pm 81$ & 0.85 & & 508 & & 466 & 715 & 1996-13 & 165 & $\begin{array}{l}\text { WGMS database, ERA- } \\
\text { Interim }\end{array}$ & $\begin{array}{l}\text { Inst. Geography, } \\
\text { Torun }\end{array}$ & 12,36 \\
\hline Kongsvegen & $78^{\circ} 48^{\prime} \mathrm{N}$ & $12^{\circ} 59^{\prime} \mathrm{E}$ & $547 \pm 84$ & 0.29 & & 466 & & 685 & 835 & 1987-14 & 165 & $\begin{array}{l}36,37,38,39,40,50,51 \\
52,53,77\end{array}$ & $\mathrm{NPI}$ & 12,36 \\
\hline Hansbreen & $77^{\circ} 05^{\prime} \mathrm{N}$ & $15^{\circ} 40^{\prime} \mathrm{E}$ & $355 \pm 77$ & 1.61 & & 592 & & 967 & 1117 & 1989-14 & 157 & $\begin{array}{c}41,42,43,44,45,46,47 \\
48,49,50,51,52,77\end{array}$ & PAS & 12,36 \\
\hline
\end{tabular}




\begin{tabular}{|c|c|c|c|c|c|c|c|c|c|c|c|c|c|c|}
\hline Glacier & Geographic & Coordinates & ELA m a.s.I. & $\begin{array}{l}\mathrm{T}_{\text {JJA }} \\
\text { ERA- } \\
\text { Interim }\end{array}$ & $\begin{array}{l}\mathrm{T}_{\text {JJA }} \\
\text { FUB. } \\
\text { Atmos. }\end{array}$ & $\begin{array}{l}\text { Ann. Precip. } \\
\text { mm, ERA- } \\
\text { Interim }\end{array}$ & $\begin{array}{l}\text { Ann. } \\
\text { Precip. } \\
\text { APGD mm }\end{array}$ & $\begin{array}{l}\text { Bw mm } \\
\text { w.e.obs. } \\
\text { at ELA }\end{array}$ & $\begin{array}{l}\text { Bw }+ \\
\text { Psummer, } \\
\text { mm }\end{array}$ & $\begin{array}{l}\text { ELA observed } \\
\text { years }\end{array}$ & $\begin{array}{l}\text { Global } \\
\text { radiation, } \\
\mathrm{W} \mathrm{m}^{-2}\end{array}$ & $\begin{array}{l}\text { Mass balance and precip. } \\
\text { data source }\end{array}$ & $\begin{array}{l}\text { Organization for } \\
\text { mass balance obs. }\end{array}$ & $\begin{array}{l}\text { Radiation } \\
\text { data source }\end{array}$ \\
\hline \multicolumn{15}{|l|}{ Alaska and Cordillera } \\
\hline Gulkana Glacier & $63^{\circ} 18^{\prime} \mathrm{N}$ & $145^{\circ} 25^{\prime} \mathrm{W}$ & $1774 \pm 73$ & 4.64 & 3.5 & 636 & & 1190 & 2540 & 1965-12 & 174 & $\begin{array}{c}5,10,20,21,23,35,36,37 \\
38,39,40,50,51,52,53\end{array}$ & USGS & 12 \\
\hline Wolverine Glacier & $60^{\circ} 24^{\prime} \mathrm{N}$ & $148^{\circ} 54^{\prime} \mathrm{W}$ & $1172 \pm 118$ & 6.08 & 5.6 & 2598 & & 1300 & 3600 & 1965-12 & 174 & $\begin{array}{c}5,10,20,21,23,35,36,37 \\
38,39,40,50,51,52,53\end{array}$ & USGS & 12 \\
\hline Taku & $58^{\circ} 33^{\prime} \mathrm{N}$ & $134^{\circ} 08^{\prime} \mathrm{W}$ & $1006 \pm 119$ & 8.75 & & 2117 & & & 2120 & 1946-13 & 183 & $\begin{array}{l}40,50,51,52,53,80, \text { ERA- } \\
\text { Interim }\end{array}$ & JIRP & 12,36 \\
\hline Lemon Creek Glacier & $58^{\circ} 23^{\prime} \mathrm{N}$ & $134^{\circ} 14^{\prime} \mathrm{W}$ & $1066 \pm 109$ & 7.48 & 7.0 & 2307 & & 1750 & 2850 & 1956-13 & 205 & $\begin{array}{l}5,10,20,21,22,23,35,36 \\
37,38,39,40,50,51,52 \\
53\end{array}$ & Michigan SU & $1,33,36$ \\
\hline Alexander Glacier & $57^{\circ} 06^{\prime} \mathrm{N}$ & $130^{\circ} 49^{\prime} \mathrm{W}$ & $1739 \pm 139$ & 4.44 & 3.7 & 1899 & & 1800 & 2050 & 1973-90 & 195 & $11,23,35$ & Glaciol. Div. & 1 \\
\hline Yuri Glacier & $56^{\circ} 58^{\prime} \mathrm{N}$ & $130^{\circ} 42^{\prime} \mathrm{W}$ & $1817 \pm 152$ & 3.96 & 3.3 & 1976 & & 1600 & 1850 & 1978-90 & 195 & $11,23,35$ & Glaciol. Div. & 1 \\
\hline Andrei Glacier & $56^{\circ} 57^{\prime} \mathrm{N}$ & $130^{\circ} 59^{\prime} \mathrm{W}$ & $1519 \pm 94$ & 5.74 & 5.1 & 2133 & & 1900 & 2150 & 1977-90 & 195 & $11,23,35$ & Glaciol. Div. & 1 \\
\hline Ram River Glacier & $51^{\circ} 51^{\prime} \mathrm{N}$ & $116^{\circ} 11^{\prime} \mathrm{W}$ & $2838 \pm 109$ & 2.95 & 4.7 & 699 & & 1000 & 1180 & 1966-75 & 245 & $3,10,72$ & Glaciol. Div. & $1,14,36$ \\
\hline Peyto Glacier & $51^{\circ} 40^{\prime} \mathrm{N}$ & $116^{\circ} 33^{\prime} \mathrm{W}$ & $2724 \pm 120$ & 3.82 & 6.1 & 766 & & 1400 & 1600 & 1966-13 & 224 & $\begin{array}{c}5,10,23,35,36,37,38,39 \\
40,50,51,52,53,72\end{array}$ & Glaciol. Div. & 15,36 \\
\hline Bench Glacier & $51^{\circ} 27^{\prime} \mathrm{N}$ & $124^{\circ} 56^{\prime} \mathrm{W}$ & 1893 & 6.8 & 6.4 & 1526 & & 2000 & 2300 & $1981-90$ & 223 & $24,35,72$ & Glaciol. Div. & 1 \\
\hline Tiedemann Glacier & $51^{\circ} 20^{\prime} \mathrm{N}$ & $125^{\circ} 00^{\prime} \mathrm{W}$ & 1954 & 6.27 & 6.2 & 1683 & & 2300 & 2550 & $1981-90$ & 223 & 24, 35, WGMS database, 72 & Glaciol. Div. & 1 \\
\hline Woolsey Glacier & $51^{\circ} 01^{\prime} \mathrm{N}$ & $118^{\circ} 12^{\prime} \mathrm{W}$ & $2268 \pm 145$ & 7.03 & 8.9 & 1214 & & 2700 & 3100 & 1965-75 & 245 & $\begin{array}{l}\text { 3, 10, WGMS database, } 72 \text {, } \\
\text { ERA-Interim }\end{array}$ & Glaciol. Div. & 1,36 \\
\hline Sykora Glacier & $50^{\circ} 53^{\prime} \mathrm{N}$ & $123^{\circ} 34^{\prime} \mathrm{W}$ & 2200 & 5.46 & 4.5 & 1444 & & 1900 & 2050 & 1976-85 & 225 & $11,23,35,72$ & Glaciol. Div. & 1,36 \\
\hline Bridge Glacier & $50^{\circ} 49^{\prime} \mathrm{N}$ & $123^{\circ} 33^{\prime} \mathrm{W}$ & 2200 & 5.39 & 4.5 & 1503 & & 1950 & 2100 & 1976-85 & 225 & $11,23,35,72$ & Glaciol. Div. & 1,36 \\
\hline Zavisha Glacier & $50^{\circ} 48^{\prime} \mathrm{N}$ & $123^{\circ} 25^{\prime} \mathrm{W}$ & 2280 & 4.9 & 4.1 & 1451 & & 1750 & 1900 & 1976-85 & 225 & $11,23,35,72$ & Glaciol. Div. & 1,36 \\
\hline Place Glacier & $50^{\circ} 16^{\prime} \mathrm{N}$ & $122^{\circ} 36^{\prime} \mathrm{W}$ & $2257 \pm 191$ & 5.21 & 6.8 & 1601 & & 2100 & 2240 & 1965-13 & 242 & $\begin{array}{c}5,10,23,35,36,37,38,39 \\
40,50,51,52,53,72\end{array}$ & Glaciol. Div. & 1,16 \\
\hline Helm Glacier & $49^{\circ} 58^{\prime} \mathrm{N}$ & $123^{\circ} 00^{\prime} \mathrm{W}$ & $2082 \pm 98$ & 5.98 & 5.0 & 2206 & & 2100 & 2350 & 1976-13 & 225 & $\begin{array}{c}11,23,35,36,37,38,39 \\
40,50,51,52,53,72\end{array}$ & Glaciol. Div. & 1,36 \\
\hline Sentinel Glacier & $49^{\circ} 54^{\prime} \mathrm{N}$ & $122^{\circ} 59^{\prime} \mathrm{W}$ & $1828 \pm 126$ & 7.51 & 8.7 & 2150 & & 3500 & 3750 & 1966-85 & 233 & $\begin{array}{l}5,10,23,35,72, \text { ERA- } \\
\text { Interim }\end{array}$ & Glaciol. Div. & 1,17 \\
\hline $\begin{array}{l}\text { South Cascade } \\
\text { Glacier }\end{array}$ & $48^{\circ} 45^{\prime} \mathrm{N}$ & $121^{\circ} 03^{\prime} \mathrm{W}$ & $1954 \pm 113$ & 8.02 & 6.9 & 1406 & & 2770 & 3020 & 1964-11 & 245 & $\begin{array}{r}5,23,35,36,37,38,39,40 \\
50,51,52,53, \text { ERA-Interim }\end{array}$ & USGS & $1,12,36$ \\
\hline Nisqually Glacier & $46^{\circ} 08^{\prime} \mathrm{N}$ & $121^{\circ} 44^{\prime} \mathrm{W}$ & 2590 & 4.28 & 3.9 & 1187 & & 3070 & 3320 & $\begin{array}{l}\text { 1967-68/ } \\
06-13\end{array}$ & 260 & $\begin{array}{l}\text { 5, WGMS database, ERA- } \\
\text { Interim }\end{array}$ & USGS & 1,17 \\
\hline \multicolumn{15}{|l|}{ Labrador } \\
\hline Superguksoak & $58^{\circ} 57^{\prime} \mathrm{N}$ & $63^{\circ} 47^{\prime} \mathrm{W}$ & 913 & 4.94 & & 770 & & & 770 & 1982-84 & 190 & 35, ERA-Interim & $\begin{array}{l}\text { Memorial } \\
\text { University }\end{array}$ & 1,35 \\
\hline Hidden & $58^{\circ} 56^{\prime} \mathrm{N}$ & $63^{\circ} 33^{\prime} \mathrm{W}$ & 1033 & 4.57 & & 764 & & & 595 & 1982-84 & 190 & 35, ERA-Interim & $\begin{array}{l}\text { Memorial } \\
\text { University }\end{array}$ & 1,35 \\
\hline Abraham & $58^{\circ} 56^{\prime} \mathrm{N}$ & $63^{\circ} 32^{\prime} \mathrm{W}$ & 983 & 4.73 & & 763 & & & 600 & 1982-84 & 190 & 35, ERA-Interim & $\begin{array}{l}\text { Memorial } \\
\text { University }\end{array}$ & 1,35 \\
\hline Minaret & $58^{\circ} 53^{\prime} \mathrm{N}$ & $63^{\circ} 41^{\prime} \mathrm{W}$ & 1290 & 3.69 & & 768 & & & 600 & 1982-84 & 183 & 35, ERA-Interim & $\begin{array}{l}\text { Memorial } \\
\text { University }\end{array}$ & 1,35 \\
\hline
\end{tabular}




\begin{tabular}{|c|c|c|c|c|c|c|c|c|c|c|c|c|c|c|}
\hline Kårsojietna & $68^{\circ} 21^{\prime} \mathrm{N}$ & $18^{\circ} 19^{\prime} \mathrm{E}$ & $1092 \pm 87$ & 6.11 & & 1030 & & 1888 & 2460 & 1982-93 & 200 & $35,36,37,57$ & $\begin{array}{l}\text { Stockholm } \\
\text { University }\end{array}$ & 36 \\
\hline Blåisen & $68^{\circ} 20^{\prime} \mathrm{N}$ & $17^{\circ} 51^{\prime} \mathrm{E}$ & 1063 & 6.22 & 4.6 & 1101 & & 1700 & 1900 & 1963-68 & 180 & 5,54 & NWREB & 12,36 \\
\hline Storsteinfjellbreen & $68^{\circ} 13^{\prime} \mathrm{N}$ & $17^{\circ} 55^{\prime} \mathrm{E}$ & 1317 & 4.71 & 3.1 & 1082 & & 1350 & 1600 & $\begin{array}{r}1964-68 / \\
1991-95\end{array}$ & 180 & $5,37,54$, ERA-Interim & NWREB & 12,36 \\
\hline Storglaciären & $67^{\circ} 54^{\prime} \mathrm{N}$ & $18^{\circ} 34^{\prime} \mathrm{E}$ & $1489 \pm 66$ & 3.96 & 3.8 & 924 & & 1320 & 1720 & 1959-14 & 180 & $\begin{array}{l}5,10,23,35,36,37,38,39 \\
40,50,51,52,53, \text { ERA- } \\
\text { Interim }\end{array}$ & $\begin{array}{l}\text { Stockholm } \\
\text { University }\end{array}$ & 12,36 \\
\hline Trollbergdalsbreen & $66^{\circ} 43^{\prime} \mathrm{N}$ & $14^{\circ} 27^{\prime} \mathrm{E}$ & 1089 & 6.33 & 5.6 & 1376 & & 2950 & 3200 & $\begin{array}{r}1970-75 / \\
1990-94\end{array}$ & 210 & 10,37 & NWREB & 12,36 \\
\hline Engabreen & $66^{\circ} 39^{\prime} \mathrm{N}$ & $13^{\circ} 51^{\prime} \mathrm{E}$ & $1114 \pm 165$ & 6.12 & 6.3 & 1391 & & 2700 & 3050 & 1969-14 & 210 & $\begin{array}{l}5,10,23,35,36,37,38,39 \\
40,50,51,52,53, \text { ERA- } \\
\text { Interim }\end{array}$ & NWREB & 12,36 \\
\hline Høgtuvbreen & $66^{\circ} 27^{\prime} \mathrm{N}$ & $13^{\circ} 39^{\prime} \mathrm{E}$ & 845 & 7.73 & 7.7 & 1409 & & 3450 & 3850 & 1971-77 & 225 & $10,23,54$ & NWREB & 12,36 \\
\hline Alfotbreen & $61^{\circ} 45^{\prime} \mathrm{N}$ & $5^{\circ} 39^{\prime} \mathrm{E}$ & $1168 \pm 182$ & 6.74 & 6.8 & 1797 & & 3450 & 3770 & $1964-14$ & 225 & $\begin{array}{l}5,10,23,35,36,37,38,39 \\
40,50,51,52,53, \text { ERA- } \\
\text { Interim }\end{array}$ & NWREB & 12,36 \\
\hline Nigårdsbreen & $61^{\circ} 43^{\prime} \mathrm{N}$ & $7^{\circ} 08^{\prime} \mathrm{E}$ & $1510 \pm 179$ & 5.23 & 4.9 & 1666 & & 2000 & 2300 & $1964-14$ & 180 & $\begin{array}{l}5,10,23,35,36,37,38,39 \\
40,50,51,52,53 \\
\text { Interim }\end{array}$ & NWREB & 12,36 \\
\hline Gråsubreen & $61^{\circ} 39^{\prime} \mathrm{N}$ & $8^{\circ} 36^{\prime} \mathrm{E}$ & $2163 \pm 159$ & 1.42 & 1.9 & 1126 & & 750 & 1000 & 1964-12 & 180 & $\begin{array}{l}5,10,23,35,36,37,38,39 \\
40,50,51,52,53, \text { ERA- } \\
\text { Interim }\end{array}$ & NWREB & 12,36 \\
\hline Hellstungubreen & $61^{\circ} 34^{\prime} \mathrm{N}$ & $8^{\circ} 26^{\prime} \mathrm{E}$ & $1932 \pm 139$ & 2.89 & 2.9 & 1161 & & 1200 & 1450 & 1964-14 & 180 & $\begin{array}{l}5,10,23,35,36,37,38,39 \\
40,50,51,52,53, \text { ERA- } \\
\text { Interim }\end{array}$ & NWREB & 12,36 \\
\hline Storbreen & $61^{\circ} 34^{\prime} \mathrm{N}$ & $8^{\circ} 08^{\prime} \mathrm{E}$ & $1785 \pm 133$ & 3.83 & & 1251 & & 1437 & 2100 & $1946-14$ & 180 & $\begin{array}{l}5,10,23,35,36,37,38,39 \\
40,50,51,52,53, \text { ERA- } \\
\text { Interim }\end{array}$ & NWREB & 12,36 \\
\hline Tunsbergdalsbreen & $61^{\circ} 36^{\prime} \mathrm{N}$ & $7^{\circ} 03^{\prime} \mathrm{E}$ & 1469 & 5.54 & 5.2 & 1686 & & 2150 & 2400 & 1966-72 & 180 & $5,10,54$ & NWREB & 12,36 \\
\hline Austre Memurubre & $61^{\circ} 33^{\prime} \mathrm{N}$ & $8^{\circ} 30^{\prime} \mathrm{E}$ & 2025 & 2.31 & 2.1 & 1139 & & 1250 & 1500 & 1968-72 & 180 & 5,54 & NWREB & 12,36 \\
\hline Vestre Memurubre & $61^{\circ} 32^{\prime} \mathrm{N}$ & $8^{\circ} 27^{\prime} \mathrm{E}$ & 1942 & 2.84 & 2.3 & 1150 & & 1350 & 1600 & 1968-72 & 180 & 5, WGMS database & NWREB & 12,36 \\
\hline Vesledalsbreen & $61^{\circ} 05^{\prime} \mathrm{N}$ & $7^{\circ} 16^{\prime} \mathrm{E}$ & 1521 & 5.59 & 5.9 & 1530 & & 2300 & 2550 & $1967-72$ & 180 & $5,10,54$ & NWREB & 12,36 \\
\hline Hardangerjökulen & $60^{\circ} 33^{\prime} \mathrm{N}$ & $7^{\circ} 22^{\prime} \mathrm{E}$ & $1671 \pm 143$ & 4.66 & 3.5 & 1516 & & 1850 & 2200 & $1964-12$ & 182 & $\begin{array}{l}5,10,23,35,36,37,38,39 \\
40,50,51,52,53\end{array}$ & NWREB & 12,18 \\
\hline Folgefonni-East & $60^{\circ} 09^{\prime} \mathrm{N}$ & $6^{\circ} 29^{\prime} \mathrm{E}$ & 1435 & 5.82 & 5.7 & 1806 & & 2700 & 3150 & 1964-68 & 200 & 5 & NWREB & 12,36 \\
\hline Folgefonni-West & $60^{\circ} 09^{\prime} \mathrm{N}$ & $6^{\circ} 29^{\prime} \mathrm{E}$ & 1435 & 5.82 & 5.4 & 1806 & & 2600 & 3050 & 1964-68 & 200 & 5 & NWREB & 12,36 \\
\hline $\begin{array}{l}\text { Bondhusbreen } \\
\text { Alps }\end{array}$ & $60^{\circ} 02^{\prime} \mathrm{N}$ & $6^{\circ} 20^{\prime} \mathrm{E}$ & 1525 & 5.24 & 5.1 & 1841 & & 2400 & 2830 & $1976-80$ & 205 & 23 & NWREB & 12,36 \\
\hline Sonnblick Kees & $47^{\circ} 07^{\prime} \mathrm{N}$ & $12^{\circ} 36^{\prime} \mathrm{E}$ & $2798 \pm 130$ & 3.46 & & 1445 & 2136 & 1300 & 2025 & 1958-14 & 215 & $38,39,40,52,53$, ZAMG & DGGS & 32 \\
\hline Pasterze & $47^{\circ} 06^{\prime} \mathrm{N}$ & $12^{\circ} 42^{\prime} \mathrm{E}$ & 3023 & 1.97 & & 1449 & 2047 & & 1450 & $2005-13$ & 217 & $\begin{array}{l}\text { WGMS database, ERA- } \\
\text { Interim }\end{array}$ & ZAMG & 12,36 \\
\hline Kleinfleiss Kees & $47^{\circ} 03^{\prime} \mathrm{N}$ & $12^{\circ} 57^{\prime} \mathrm{E}$ & $2982 \pm 110$ & 2.27 & & 1460 & 1674 & & 1673 & 1999-14 & 222 & WGMS database, ZAMG & ZAMG & 32,37 \\
\hline Goldberg Kees & $47^{\circ} 02^{\prime} \mathrm{N}$ & $12^{\circ} 58^{\prime} \mathrm{E}$ & $3008 \pm 76$ & 2.12 & & 1463 & 1379 & 1750 & 2200 & $2001-13$ & 222 & 82, WGMS database, ZAMG & ZAMG & 32,37 \\
\hline Wurten Kees & $47^{\circ} 02^{\prime} \mathrm{N}$ & $13^{\circ} 00^{\prime} \mathrm{E}$ & $3009 \pm 145$ & 2.11 & & 1463 & 1608 & 1411 & 2125 & 1983-13 & 222 & $37,38,39$, ZAMG & ZAMG & 32,37 \\
\hline Vernagtferner & $46^{\circ} 52^{\prime} \mathrm{N}$ & $10^{\circ} 49^{\prime} \mathrm{E}$ & $3144 \pm 158$ & 1.41 & 2.0 & 1410 & 827 & 924 & 1574 & 1964-14 & 245 & 25,63, ZAMG & KGBAW & $\begin{array}{l}19,20,2 \\
34\end{array}$ \\
\hline Claridenfirn & $46^{\circ} 51^{\prime} \mathrm{N}$ & $8^{\circ} 54^{\prime} \mathrm{E}$ & $2771 \pm 125$ & 4 & & 1430 & & 1656 & 2945 & $2001-11$ & 215 & $\begin{array}{l}\text { 81, personal communica- } \\
\text { tion Hans Müller-Lemans }\end{array}$ & $\begin{array}{l}\text { Telgeso AG, } \\
\text { Sargabs }\end{array}$ & 36 \\
\hline
\end{tabular}




\begin{tabular}{|c|c|c|c|c|c|c|c|c|c|c|c|c|c|c|}
\hline Glacier & Geographic & Coordinates & ELA m a.s.l. & $\begin{array}{l}\mathrm{T}_{\text {JJA }} \\
\text { ERA- } \\
\text { Interim }\end{array}$ & $\begin{array}{l}\mathrm{T}_{\text {JJA }} \\
\text { FUB. } \\
\text { Atmos. }\end{array}$ & $\begin{array}{l}\text { Ann. Precip. } \\
\text { mm, ERA- } \\
\text { Interim }\end{array}$ & $\begin{array}{l}\text { Ann. } \\
\text { Precip. } \\
\text { APGD mm }\end{array}$ & $\begin{array}{l}\text { Bw mm } \\
\text { w.e.obs. } \\
\text { at ELA }\end{array}$ & $\begin{array}{l}\text { Bw }+ \\
\text { Psummer, } \\
\text { mm }\end{array}$ & $\begin{array}{l}\text { ELA observed } \\
\text { years }\end{array}$ & $\begin{array}{l}\text { Global } \\
\text { radiation, } \\
\mathrm{W} \mathrm{m}^{-2}\end{array}$ & $\begin{array}{l}\text { Mass balance and precip. } \\
\text { data source }\end{array}$ & $\begin{array}{l}\text { Organization for } \\
\text { mass balance obs. }\end{array}$ & $\begin{array}{l}\text { Radiation } \\
\text { data source }\end{array}$ \\
\hline Hintereisferner & $46^{\circ} 48^{\prime} \mathrm{N}$ & $10^{\circ} 46^{\prime} \mathrm{E}$ & $3078 \pm 218$ & 1.9 & 2.0 & 1402 & 790 & 1500 & 2100 & 1953-14 & 265 & 26,27, ZAMG & IMGUI & $18,22,23$ \\
\hline Rhonegletscher & $46^{\circ} 37^{\prime} \mathrm{N}$ & $8^{\circ} 24^{\prime} \mathrm{E}$ & $2895 \pm 92$ & 3.17 & 2.2 & 1323 & 1945 & 1950 & 2230 & $\begin{array}{l}1885-09 / 80- \\
83 / 13-14\end{array}$ & 250 & $\begin{array}{l}\text { 28, WGMS database, } \\
\text { MeteoSwiss }\end{array}$ & VAW & 24 \\
\hline Aletschgletscher & $46^{\circ} 30^{\prime} \mathrm{N}$ & $8^{\circ} 02^{\prime} \mathrm{E}$ & 2923 & 3.1 & 2.1 & 1340 & 2087 & & 3000 & 1865-2006 & 257 & 65, MeteoSwiss & VAW & 36 \\
\hline Careser & $46^{\circ} 27^{\prime} \mathrm{N}$ & $10^{\circ} 41^{\prime} \mathrm{E}$ & $3330 \pm 184$ & 1.57 & 1.6 & 1356 & 893 & 1189 & 1450 & $1966-14$ & 255 & $\begin{array}{l}5,10,23,35,36,37,38,39 \\
40,50,51,52,53, \text { ERA- } \\
\text { Interim }\end{array}$ & Univ. Padua & 12 \\
\hline Griesgletscher & $46^{\circ} 26^{\prime} \mathrm{N}$ & $8^{\circ} 20^{\prime} \mathrm{E}$ & $3004 \pm 149$ & 2.7 & 2.7 & 1281 & 1761 & 1500 & 2250 & $1962-14$ & 250 & 29, 66, MeteoSwiss & VAW & 18 \\
\hline Basodino & $46^{\circ} 25^{\prime} \mathrm{N}$ & $8^{\circ} 29^{\prime} \mathrm{E}$ & $2985 \pm 168$ & 2.79 & & 1271 & 1668 & 1421 & 3040 & $1992-14$ & 265 & $\begin{array}{l}\text { 38, 39, 40, WGMS data- } \\
\text { base, G. Kappenberger, } \\
\text { MeteoSwiss }\end{array}$ & G. Kappenberger & 36 \\
\hline Findelengletscher & $46^{\circ} 00^{\prime} \mathrm{N}$ & $7^{\circ} 52^{\prime} \mathrm{E}$ & 3257 & 1.31 & & 1224 & 1579 & & 2610 & 2005-13 & 275 & $\begin{array}{l}\text { WGMS database, } \\
\text { MeteoSwiss }\end{array}$ & VAW & 36 \\
\hline Argentiere & $45^{\circ} 57^{\prime} \mathrm{N}$ & $6^{\circ} 59^{\prime} \mathrm{E}$ & 2859 & 3.8 & & 1269 & 1888 & & 1900 & 2001-05 & 245 & WGMS database & CNRS & 36 \\
\hline Marmolada & $44^{\circ} 07^{\prime} \mathrm{N}$ & $7^{\circ} 23^{\prime} \mathrm{E}$ & 2740 & 6.28 & 4.3 & 878 & 1437 & 1050 & 1250 & 1964-66 & 220 & 5,74 & Univ. Padua & 12,18 \\
\hline Calderone & $42^{\circ} 28^{\prime} \mathrm{N}$ & $13^{\circ} 37^{\prime} \mathrm{E}$ & $2713 \pm 153$ & 6.76 & & 804 & & 2575 & 2925 & 1995-13 & 242 & 39,40, WGMS database, 70 & IMONT & 12,36 \\
\hline \multicolumn{15}{|l|}{ Pyrenees } \\
\hline Maladeta & $42^{\circ} 39^{\prime} \mathrm{N}$ & 038'E & $3112 \pm 71$ & 4.26 & & 845 & & 2036 & 2558 & 1992-11 & 281 & $\begin{array}{l}37,38,39,40,50,51,52 \\
53,75\end{array}$ & 175SA \& MMA & 12,36 \\
\hline \multicolumn{15}{|l|}{ Caucasus } \\
\hline Dzankuat & $43^{\circ} 12^{\prime} \mathrm{N}$ & $42^{\circ} 44^{\prime} \mathrm{E}$ & $3220 \pm 104$ & 4.53 & 4.1 & 1245 & & 2250 & 2800 & 1968-11 & 265 & 23, ERA-Interim & $\begin{array}{l}\text { Moscow State } \\
\text { University }\end{array}$ & 12,36 \\
\hline \multicolumn{15}{|l|}{ Tienshan and Altai } \\
\hline $\begin{array}{l}\text { Tsentralniy } \\
\text { Tuyuksuyskiy }\end{array}$ & $43^{\circ} 03^{\prime} \mathrm{N}$ & $77^{\circ} 05^{\prime} \mathrm{E}$ & $3826 \pm 118$ & 1.51 & 3.6 & 838 & & 760 & 1160 & 1957-14 & 280 & 5, 10, 23, ERA-Interim & ASKASSR & 12,36 \\
\hline $\begin{array}{l}\text { No. } 1 \text { Glacier } \\
\text { Urumqi }\end{array}$ & $43^{\circ} 07^{\prime} \mathrm{N}$ & $86^{\circ} 49^{\prime} \mathrm{E}$ & $4068 \pm 92$ & -0.04 & -0.2 & 614 & & 160 & 520 & 1959-12 & 250 & 10, WGMS, LIGG & LIGG & 25,26 \\
\hline \multicolumn{15}{|l|}{ Qilianshan } \\
\hline Qiyi & $39^{\circ} 23^{\prime} \mathrm{N}$ & $96^{\circ} 59^{\prime} \mathrm{E}$ & 4626 & -0.64 & & 255 & & 350 & 650 & 1975-85 & 265 & $\begin{array}{l}\text { WGMS database, } 68,69, \\
78,79\end{array}$ & & 33,36 \\
\hline $\begin{array}{l}\text { Shuiguanhe No. } 4 \\
\text { Pamir and Himalaya }\end{array}$ & $37^{\circ} 33^{\prime} \mathrm{N}$ & \multicolumn{10}{|c|}{ Pamir and Himalaya } & WGMS database, 68, 69 & & 33,36 \\
\hline Abramov & $39^{\circ} 38^{\prime} \mathrm{N}$ & $71^{\circ} 36^{\prime} \mathrm{E}$ & $4230 \pm 98$ & 0.85 & & 1127 & & 1393 & 1715 & $\begin{array}{r}1968-98 / \\
2012-14\end{array}$ & 305 & WGMS database, 83 & CAIAG & 33,36 \\
\hline Rikha Samba & $28^{\circ} 49^{\prime} \mathrm{N}$ & $83^{\circ} 30^{\prime} \mathrm{E}$ & 5737 & -1.3 & -1.3 & 1580 & & 20 & 1580 & $\begin{array}{c}1974 / 99 / \\
12-13\end{array}$ & 245 & $\begin{array}{l}\text { 30, } 62, \text { WGMS database, } \\
\text { ERA-Interim }\end{array}$ & WRI & 27,30 \\
\hline \multicolumn{15}{|l|}{ South America } \\
\hline Chacaltaya & $16^{\circ} 21^{\prime} \mathrm{S}$ & $68^{\circ} 07^{\prime} \mathrm{W}$ & $5441 \pm 83$ & -3.69 & & 2982 & & 18 & 1475 & $1992-12$ & 207 & 38,39 & $\mathrm{IHH}$ & 33 \\
\hline Glaciar de Los Tres & $49^{\circ} 20^{\prime} \mathrm{S}$ & $73^{\circ} 00^{\prime} \mathrm{W}$ & $1430 \pm 17$ & 1.92 & & 1766 & & 2219 & 2775 & 1996-98 & 300 & 38, WGMS database & $\begin{array}{l}\text { Moscow State } \\
\text { University }\end{array}$ & 33 \\
\hline Hodges Glacier & $54^{\circ} 17^{\prime} \mathrm{S}$ & $36^{\circ} 30^{\prime} \mathrm{W}$ & 435 & 1.06 & 1.9 & 892 & & 1480 & 1850 & $1951-60$ & 240 & 33, ERA-Interim & Univ. East Anglia & 29 \\
\hline Martial Este & $54^{\circ} 47^{\prime} \mathrm{S}$ & $68^{\circ} 24^{\prime} \mathrm{W}$ & $1082 \pm 42$ & 1.48 & & 1051 & & 960 & 1300 & $2001-13$ & 280 & $39,50,51,52,53$ & UNC & 33 \\
\hline \multicolumn{15}{|l|}{ New Zealand } \\
\hline $\begin{array}{l}\text { Tasman Glaceir } \\
\text { The Antarctic }\end{array}$ & $43^{\circ} 30^{\prime} \mathrm{S}$ & $170^{\circ} 20^{\prime} \mathrm{E}$ & $1887 \pm 176$ & 5.62 & 7.7 & 1716 & & 2500 & 3250 & 1966-75 & 250 & 5, 33, ERA-Interim & Ministry of works & 12,36 \\
\hline
\end{tabular}




\begin{tabular}{|c|c|c|c|c|c|c|c|c|c|c|c|c|c|}
\hline Johnsons & $62^{\circ} 40^{\prime} \mathrm{S}$ & $60^{\circ} 21^{\prime} \mathrm{W}$ & 179 & 1.23 & & 893 & 761 & 1004 & 2002-13 & 225 & WGMS database & UPM & 33 \\
\hline Hurd & $62^{\circ} 41^{\prime} \mathrm{S}$ & $60^{\circ} 24^{\prime} \mathrm{W}$ & 203 & 1.23 & & 897 & 624 & 867 & $2002-13$ & 225 & WGMS database & UPM & 33 \\
\hline $\begin{array}{l}\text { Glacier G1, } \\
\text { Deception Island }\end{array}$ & $63^{\circ} 00^{\prime} \mathrm{S}$ & $60^{\circ} 40^{\prime} \mathrm{W}$ & 325 & 0.15 & 0.0 & 947 & 600 & 780 & 1968-71 & 220 & 5, ERA-Interim & $\begin{array}{c}\text { Ohio State } \\
\text { University }\end{array}$ & 12 \\
\hline Law Dome & $66^{\circ} 00^{\prime} \mathrm{S}$ & $112^{\circ} 00^{\prime} \mathrm{E}$ & 150 & -6.5 & -2.6 & 539 & 330 & 360 & Stratigraphic & 233 & 34, ERA-Interim & ANARE & 30,31 \\
\hline
\end{tabular}

Data sources for mass balance and precipitation.

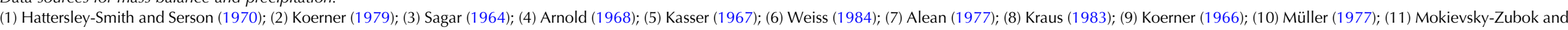

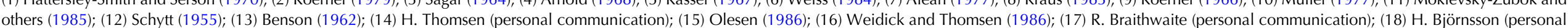

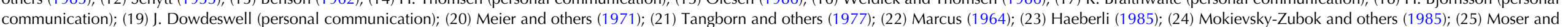

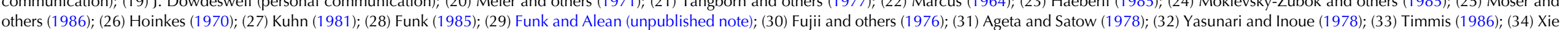

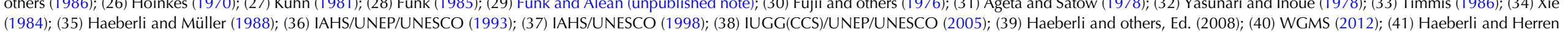

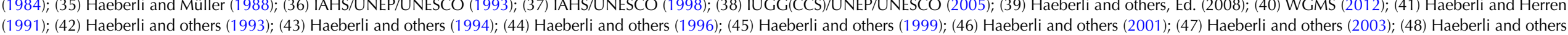

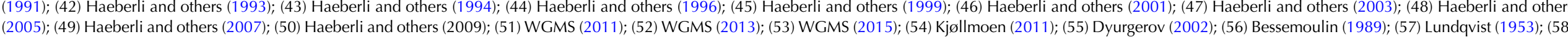

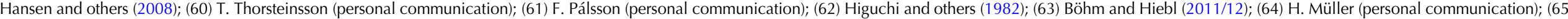

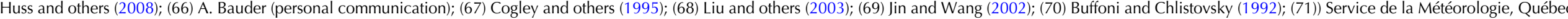

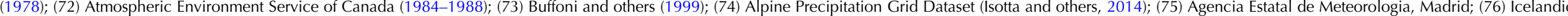

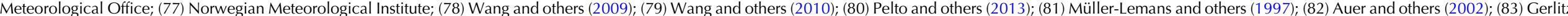
and others (2016).

Data sources for solar global radiation.

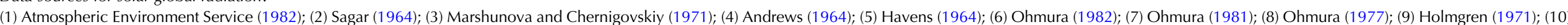

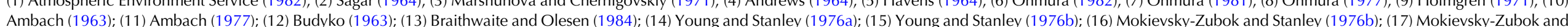

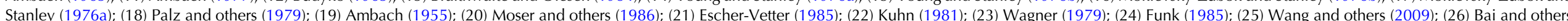

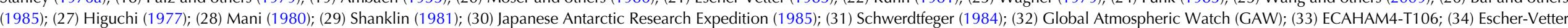
(1985); (35) Service de la Météorologie, Québec (1978); (36) Wild and others (2017); (37) Auer and others (2002).

Abbreviations for the organizations carrying out the mass balance observations.

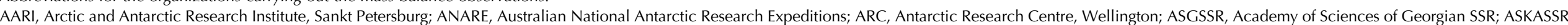

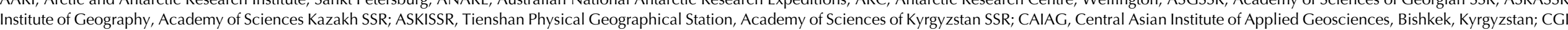

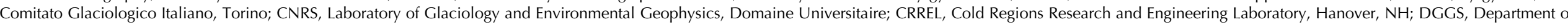

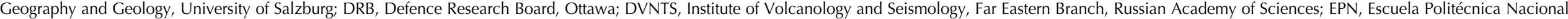

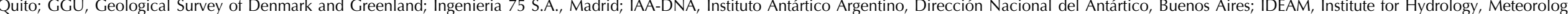

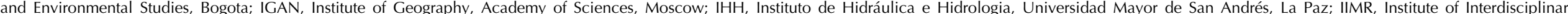

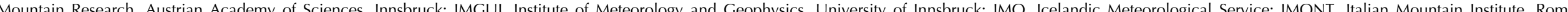
(IIGG, Lanzhou Institute of Glaciology and Geocryology; MMA, Dirección General del Agua, Ministerio de Medio Ambiente, Madrid; MSC, Meteorological Service of

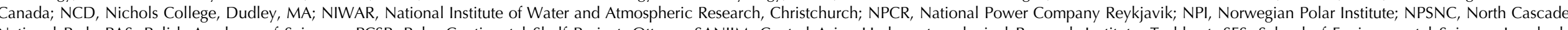

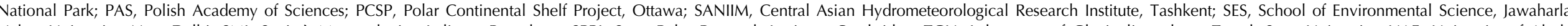

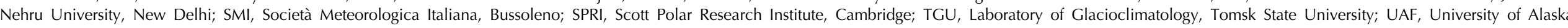

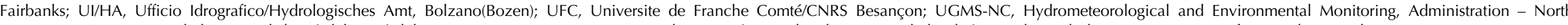

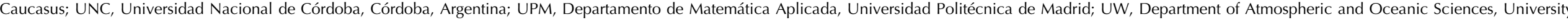

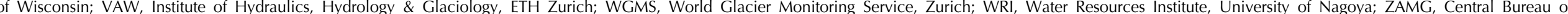
Meteorology and Geodynamics, Vienna. 


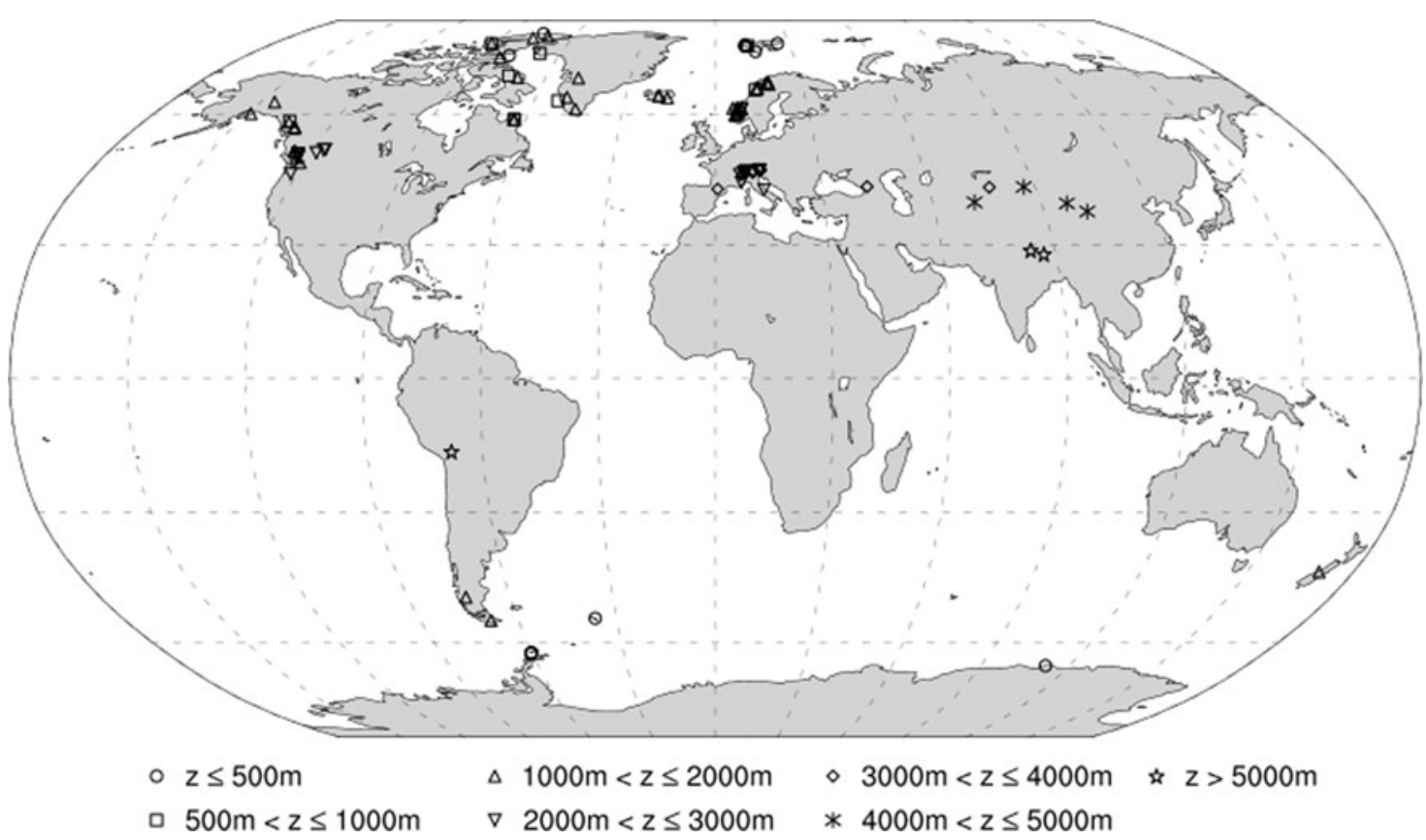

Fig. 1. Geographic distribution of the locations and the altitudes of equilibrium lines of the glaciers used in the present work.

during the melt period. The actually observed mean emission is $309 \mathrm{~W} \mathrm{~m}^{-2}$ with a maximum of $316 \mathrm{~W} \mathrm{~m}^{-2}$ (Ward Hunt Ice Shelf and Blue Glacier) and a minimum of $301 \mathrm{Wm}^{-2}$ (Vernagtferner). The mean emission is slightly smaller than the expected value from the blackbody emission at the melting point, as the glacier surface often re-freezes at night, allowing the surface to cool below the freezing point (Ambach, 1955). Although the surface emission is numerically the largest component of the energy balance, it is close to a constant. Therefore, among the fluxes of the heat sink, the latent heat of melt is the largest variable (mean of $91 \%$ of the available heat source), with two exceptions that show relatively large evapo-sublimation (Urumqi No.1 Glacier, $18 \%$ and Zongo Glacier, $60 \%$ ). These are nevertheless amazingly similar features, when one considers the diverse climatic conditions under which these glaciers are located. This situation allows the following discussion in terms of the mean fluxes that are presented in the bottom line of Table 3. The mean energy balance on the ELA takes the following form:

$$
S(1-\mathrm{a})+L \downarrow+\sigma T_{o}^{4}+H+L_{\mathrm{v}} E+C=M,
$$

where $S$ is the solar global radiation, $a$ is the albedo at ELA, $L \downarrow$ is the longwave incoming radiation, $\sigma$ is the StefanBoltzmann constant $\left(5.67 \times 10^{-8} \mathrm{~W} \mathrm{~m}^{-2} \mathrm{~K}^{-4}\right), T_{\mathrm{o}}$ is the glacier surface temperature at ELA, $H$ is the sensible heat flux from the atmosphere, $L_{v}$ is the latent heat of vaporization, $E$ is the evaporation/sublimation rate, $C$ is the subsurface energy flux mainly constituted by heat conduction and ice-internal absorption of solar radiation and $M$ is the heat used for melt. The sign is taken positive when the flux is directed to the surface. Table 3 shows that longwave incoming radiation from the atmosphere is by far the most

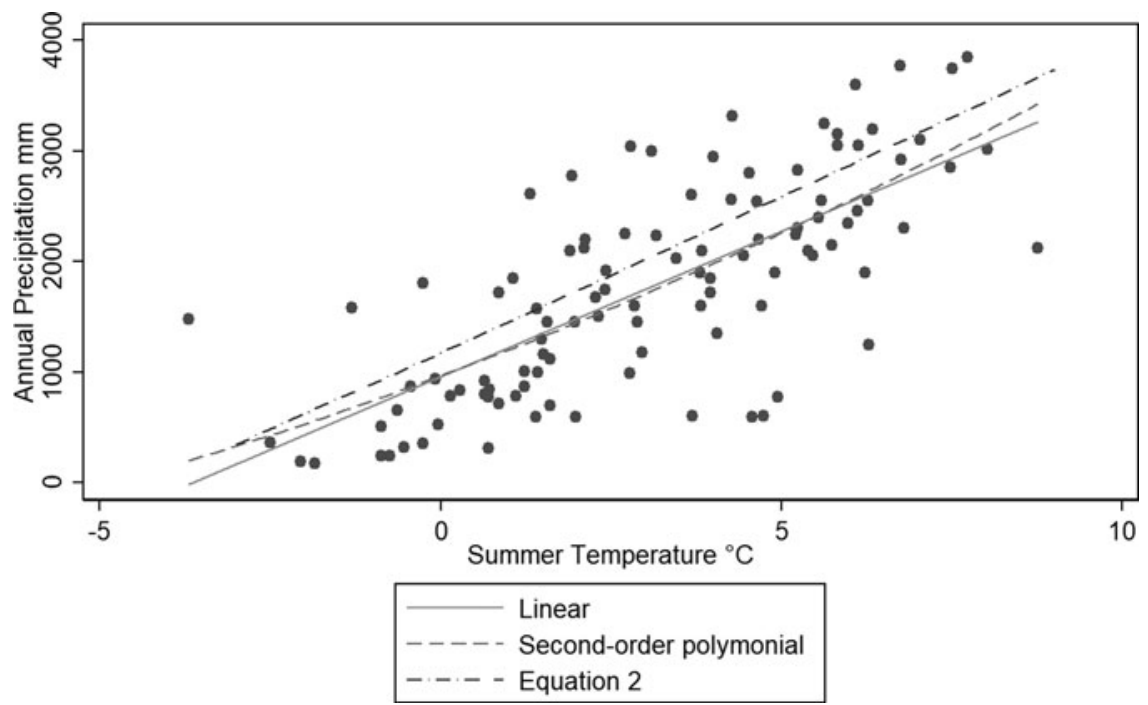

Fig. 2. Annual precipitation and summer (June, July, August or December January, February) mean air temperature at the equilibrium line altitude (ELA): $R^{2}$ is 0.63 . Two thin lines are linear and quadratic regression lines, while the dash and dot line is Eqn 2 discussed in Section 3.3 . 

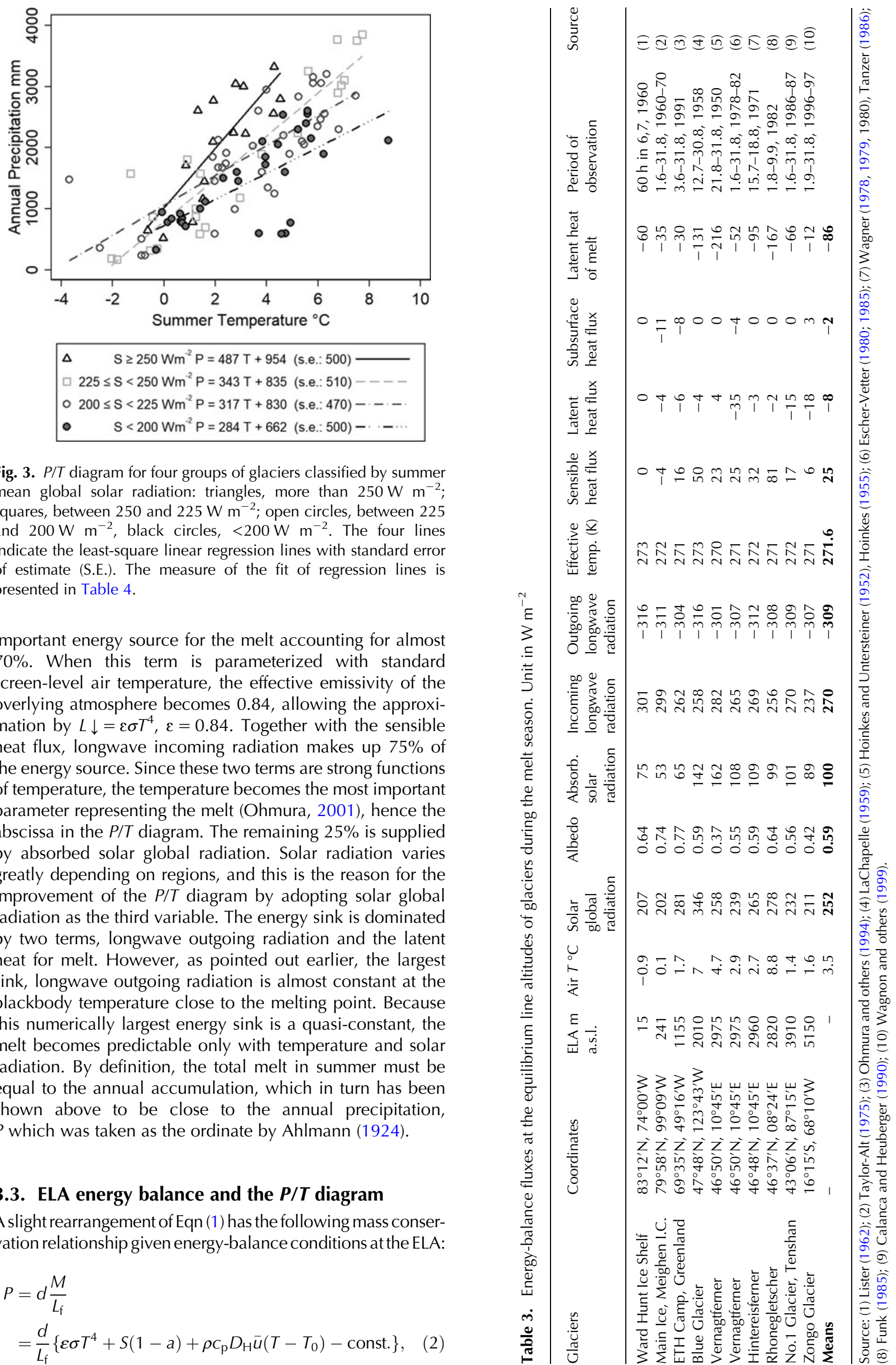

Fig. 3. $P / T$ diagram for four groups of glaciers classified by summer mean global solar radiation: triangles, more than $250 \mathrm{~W} \mathrm{~m}^{-2}$. squares, between 250 and $225 \mathrm{~W} \mathrm{~m}^{-2}$; open circles, between 225 and $200 \mathrm{~W} \mathrm{~m}^{-2}$, black circles, $<200 \mathrm{~W} \mathrm{~m} \mathrm{~m}^{-2}$. The four lines indicate the least-square linear regression lines with standard error of estimate (S.E.). The measure of the fit of regression lines is presented in Table 4.

important energy source for the melt accounting for almost $70 \%$. When this term is parameterized with standard screen-level air temperature, the effective emissivity of the overlying atmosphere becomes 0.84 , allowing the approximation by $L \downarrow=\varepsilon \sigma T^{4}, \varepsilon=0.84$. Together with the sensible heat flux, longwave incoming radiation makes up $75 \%$ of the energy source. Since these two terms are strong functions of temperature, the temperature becomes the most important parameter representing the melt (Ohmura, 2001), hence the abscissa in the $P / T$ diagram. The remaining $25 \%$ is supplied by absorbed solar global radiation. Solar radiation varies greatly depending on regions, and this is the reason for the improvement of the $P / T$ diagram by adopting solar global radiation as the third variable. The energy sink is dominated by two terms, longwave outgoing radiation and the latent heat for melt. However, as pointed out earlier, the largest sink, longwave outgoing radiation is almost constant at the blackbody temperature close to the melting point. Because this numerically largest energy sink is a quasi-constant, the melt becomes predictable only with temperature and solar radiation. By definition, the total melt in summer must be equal to the annual accumulation, which in turn has been shown above to be close to the annual precipitation, $P$ which was taken as the ordinate by Ahlmann (1924).

\subsection{ELA energy balance and the $P / T$ diagram}

A slight rearrangement of Eqn (1) has the following mass conservation relationship given energy-balance conditions at the ELA:

$$
\begin{aligned}
P & =d \frac{M}{L_{\mathrm{f}}} \\
& =\frac{d}{L_{\mathrm{f}}}\left\{\varepsilon \sigma T^{4}+S(1-a)+\rho c_{\mathrm{p}} D_{\mathrm{H}} \bar{u}\left(T-T_{0}\right)-\text { const. }\right\},
\end{aligned}
$$


where $P$ is the annual precipitation, hence summer total melt, $d$ is the duration of the melt period, in the present case the three summer months, that is, $92 \mathrm{~d}$ (90 d for the Southern Hemisphere); $L_{f}$ is the latent heat of fusion, $3.34 \times 10^{5} \mathrm{~J} \mathrm{~kg}^{-1}$, $\rho$ is the density of the atmosphere, close to $1 \mathrm{~kg} \mathrm{~m}^{-3} ; c_{\mathrm{p}}$ is the specific heat of air under constant pressure, $1004.67 \mathrm{~J} \mathrm{~kg}^{-1}$ $\mathrm{K}^{-1} ; D_{\mathrm{H}}$ is the drag coefficient for heat, close to 0.0025 for wind speed at $10 \mathrm{~m}$ above the surface (Priestley, 1959), $\bar{u}$ is the wind-speed, $T$ is the air temperature, $T_{0}$ is the surface temperature, while 'const.' is the sum of the surface radiative emission, evapo-/sublimation and sub-surface heat flux, and 315 $\mathrm{W} \mathrm{m}^{-2}$.

The numerical accuracy of Eqn (2) is tested against the observations of $P$ and $T$ for 104 glaciers presented in Table 2. The necessary values are presented in the previous paragraph and also in Table 2. The estimation of the sensible heat flux poses a slight problem, as this flux was not measured on most glaciers presented in Table 2. When wind was measured by glaciologists, often it was carried out not at the meteorological standard height of $10 \mathrm{~m}$ above the surface. The drag coefficient in micro-meteorology is traditionally referred to $10 \mathrm{~m}$. Rather than estimating the drag coefficient and wind speed separately, the authors took a direct way to estimate $\rho c_{\mathrm{p}} D_{\mathrm{H}} \bar{u}$ by regressing sensible heat flux $H$ against $\left(T-T_{0}\right)$, as is presented in Figure 4 . The gradient of the regression line is $\rho c_{\mathrm{p}} D_{\mathrm{H}} \bar{u}$ with numerical value of $7.9 \mathrm{~W} \mathrm{~m} \mathrm{~m}^{-2} \mathrm{~K}^{-1} \cdot R^{2}$ is 0.84 . This value gives the approximate value of $D_{\mathrm{H}}$ as 0.003 , which is close to 0.0025 given by Priestley (1959) and Brutsaert (2005).

Equation (2) is plotted in Figure 2, together with the empirically obtained $P / T$ relationships. There is an overestimation of $P$ by $270 \mathrm{~mm}$, equivalent to $11 \mathrm{~W} \mathrm{~m}^{-2}$ between Eqn (2)

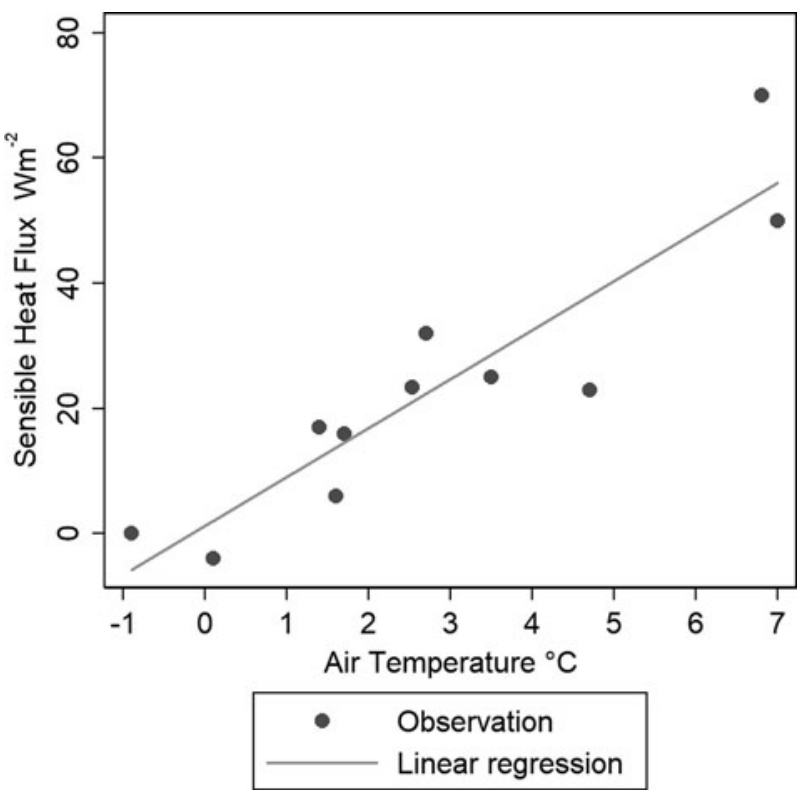

Fig. 4. Relationship between sensible heat flux and the temperature difference between the surface and the atmosphere at the equilibrium line of glaciers during the melt season, obtained based on ten experiments on nine glaciers summarized in Table 3. The gradient of the regression line is $\rho c_{\mathrm{p}} D_{\mathrm{H}} \bar{u}$ with the numerical value of $7.9 \mathrm{Wm}^{-2} \mathrm{~K}^{-1}$. $R^{2}$ is 0.84 . When detailed information such as the surface roughness length and the wind speed at the WMO standard height, that is $10 \mathrm{~m}$ above the surface, are not available, this relationship allows an order of magnitude estimation of the sensible heat flux on the glacier. and the best-fit empirical curves of 104 glaciers, which is small in comparison with the possible errors associated with the energy flux measurements. The difference may also stem from the possibility that the nine glaciers used for the derivation of Eqn (2) tended to have smaller evaporation than the mean condition of the majority of glaciers. In the present work, evaporation is not numerically treated, as it is small. It is included in the 'constant'. However, if evaporation is larger, it must be treated as an independent term, and tends to pull the theoretical line downward, as postulated by Loewe (1971). This sampling problem can be reconciled, if further energy-balance observations are carried out on the glaciers representing mean climatic conditions.

\section{DISCUSSION AND CONCLUSION}

In summary, Eqn 2, the water-equivalent transformation of the energy-balance equation, is the $\mathrm{P} / \mathrm{T}$ diagram originally proposed by Ahlmann (1924). When annual precipitation $P$ is made a dependent variable, it becomes a fourth-order polynomial in $T$, summer air temperature. The Ahlmann's $P / T$ curve expressing the climatic conditions at the ELA is a concise expression of the conservation principle. The mathematical shape of the $P / T$ curve, which was earlier speculated to take various functional forms, is a polynomial of the fourth order that is derived from both the StefanBoltzmann equation and the difference approximation of turbulent sensible heat flux.

However, the narrow temperature range of the melt season at the ELA occupying only $10^{\circ} \mathrm{C}$ between -2 and $+8^{\circ} \mathrm{C}$ offers a practical justification for approximating the $P / T$ relationship with a lower order such as a quadratic or even a linear function, which offers a possibility for wide practical applications. These approximations are $P=5.87$ $T^{2}+230 T+966$ and $P=264.1 T+957$, respectively, based on the least-square method for all 104 glaciers considered in the present study. The standard error of estimate (S.E.) of both regressions is almost identical at 648 and $650 \mathrm{~mm}$, respectively. By taking solar global radiation into account, the best-fit approximation takes the following shape: $P=$ (2.2 S-130) $T+3.12 S+122$ with S.E. of $510 \mathrm{~mm}$, where $T$ $\left({ }^{\circ} \mathrm{C}\right)$ is the mean air temperature and $S\left(\mathrm{~W} \mathrm{~m}^{-2}\right)$ is the mean solar global radiation during the melt period. $P(\mathrm{~mm})$ is the annual precipitation at the ELA. The introduction of solar radiation brought about a considerable improvement over the previous temperature-only formulation. A better fit is, however, found for the four groups of glaciers that are classified by solar global radiation as presented in Table 4. Figure 3 offers regression lines and the standard error estimates for

Table 4. The best-fit linear regressions for the precipitation and temperature relationship at the equilibrium line altitudes (ELA) of glaciers, classified with respect to solar global radiation $\left(S, \mathrm{~W} \mathrm{~m}^{-2}\right)$. The coefficients are for the form of $P(\mathrm{~mm})=\mathrm{a} T\left({ }^{\circ} \mathrm{C}\right)+\mathrm{b}$, S.E. is the standard error of estimate, and $n$ is the number of the sampled glaciers

\begin{tabular}{llrlll}
\hline Range of solar radiation & $a$ & \multicolumn{1}{c}{$b$} & $R^{2}$ & S.E. & $n$ \\
\hline$S \geq 250 \mathrm{~W} \mathrm{~m}^{-2}$ & 487 & 1015 & 0.65 & 534 & 16 \\
$225 \leq S<250$ & 350 & 793 & 0.88 & 490 & 22 \\
$200 \leq S<225$ & 245 & 1058 & 0.61 & 533 & 34 \\
$S<200$ & 213 & 729 & 0.49 & 503 & 31
\end{tabular}


each group. Among them, the best fit with the smallest standard error of estimate was found for the group with solar global radiation ranging between 225 and $250 \mathrm{~W} \mathrm{~m}^{-2}$. The reason for the best performance by glaciers of this class is not clear. The $P / T$ combinations falling above these lines represent the climate of the accumulation area, while those below the lines indicate the climate of the ablation area or the area outside the glaciers.

The introduction of solar global radiation improves the standard error of estimate of the regression lines from $\sim 650$ down to $500 \mathrm{~mm}$, which corresponds to $\sim 1.2^{\circ} \mathrm{C}$ in temperature scale. Given the wide range of variety of the climate where these glaciers are located, the present results are a considerable improvement over the earlier understanding on the climate at the glacier ELA. The present findings will contribute to the systematic understanding of the equilibrium line formation and of the total meltwater available from glaciers, and to reconstructing the temperature and precipitation on glaciers of the past.

\section{ACKNOWLEDGEMENTS}

We are supported by scientific colleagues, who supplied prepublication data, or unknown publications to the authors. We thank Michael Kuhn of the University of Innsbruck; Julian Dowdeswell of Scott Polar Research Institute, Cambridge; Christian Vincent of Laboratoire de Glaciologie et Géophysique de I'Environnement, Grenoble; Ludwig Braun and Heidi Escher-Vetter of Bavarian Academy of Sciences, München; Elke Ludewig of Central Institute of Meteorology and Geodynamics, Salzburg; Ersi Kang, Zhongqin Li, and Ninglian Wang of Key Laboratory of Cryosphere and Environment, Chinese Academy of Sciences, Lanzhou; Henrik Højmark Thomsen of Geological Survey of Denmark and Greenland; Roger J. Braithwaite of Manchester University; Thorsteinn Thorsteinsson of Iceland Meteorological Office; Guðfinna Aðalgeirsdottir and Finnur Pálsson of the University of Iceland; Christoph Schär, Martin Funk and Andreas Bauder of E.T.H., Zurich; Michael Zemp of World Glacier Monitoring Service, University of Zurich; Hans Müller of Tergeso AG, Sargans; Giovanni Kappenberger of Swiss Federal Office for Meteorology and Climatology, Locarno-Monti; Jürg Alean of Zurich, and Koji Fujita of Nagoya University. We thank Tamaki Ohmura who carried out most of the statistical calculations.

\section{REFERENCES}

Ageta $Y$ and Satow K (1978) Study of the mass balance of small glaciers in Khumbu Himal during the summer monsoon season. Seppyo, 40, Special Issue, 4-11

Ahlmann HW (1922) Glaciers in Jotunheim and their physiography. Geogr. Ann., 4, 1-57

Ahlmann HW (1924) Le niveau de glaciation comme function de l'accumulation d'humidité sous forme solide. Méthode pour le calcul de l'humidité condensée dans la haute montagne et pour l'étude de la fréquence des glaciers. Geogr. Ann., 6, 223272

Alean J (1977) Zum Massenhaushalt des Baby Glaciers auf der Axel Heiberg Insel in der kanadischen Hocharktis (1959/60 bis 1975/ 76). Diplomarbeit, Eidgenössische Technische Hochschule, Zürich

Ambach W (1955) Über den nächtlichen Wärmeumsatz der gefrorenen gletscheroberfläche. Arch. Meteorol. Geophys. Bioklimatol. Ser. A, 8(4), 411-426
Ambach W (1963) Untersuchungen zum Energieumsatz in der Ablationszone des grönländischen Inlandeises (camp IV-EGIG, $\left.69^{\circ} 40^{\prime} 05^{\prime \prime} \mathrm{N}^{\prime}, 49^{\circ} 37^{\prime} 58^{\prime \prime} \mathrm{W}\right)$. Medd. Grønl., 174(4), 311pp

Ambach W (1977) Untersuchungen zum Energieumsatz in der Ablationszone des grönländischen Inlandeises. Nachtrag. Medd. Grønl., 187(5), 66pp

Ambach W (1989) Effects of climatic perturbations on the surfaceablation regime of the Greenland ice sheet, West Greenland. J. Glaciol., 35(121), 311-316

Ambach W (1993) Effects of climatic perturbations on equilibriumline altitude, West Greenland. J. Glaciol., 39(131), 5-9

Andreassen LM, Elvehøy H, Kjøllmoen B and Engeset RV (2016) Reanalysis of long-term series of glaciological and geodetic mass balance for 10 Norwegian glaciers. Cryosphere, 10, 535-552

Andrews JT (1975) Glacial systems: an approach to glaciers and their environments. Duxbury Press, North Scituate, $191 \mathrm{pp}$

Andrews RH (1964) Meteorology and heat balance of the ablation area, White Glacier, Canadian Arctic Archipelago - summer 1960 (Lower Ice Station: $79^{\circ} 26^{\prime} \mathrm{N}, 90^{\circ} 39^{\prime} \mathrm{W}, 208 \mathrm{~m}$ ). Axel Heiberg Isl. Res. Rep., Meteorol. 1, McGill Univ., 107pp

Arnold KC (1968) Determination of changes of surface height, 19571967, of Gilman Glacier, northern Ellesmere Island, Canada. (MSc thesis, McGill University), 88pp

Atmospheric Environment Service (1982) Canadian climate normals 1951-1980, Vol. 3, Environment Canada. Atmospheric Environment Service, Toronto

Atmospheric Environment Service of Canada (1984-1988) Climatic atlas of Canada, Bd. 1-5, Atmospheric Environment of Canada, Toronto

Auer I (1992) Precipitation measurements in a high Alpine region of Austria-Intercomparison of different measuring systems. WMO/ TD No. 462, 251-256

Auer I, Böhm R and Schöner W (2001) Austrian long-term climate 1767-2000. Österreichische Beiträge zu Meteorologie und Geophysik, Heft 25, Central Institute for Meteorology and Geodynamics, Vienna, $147 \mathrm{pp}$

Auer I, Böhm R, Leymüller M and Schöner W (2002) Das Klima des Sonnblicks (The Climate of Sonnblick). Österreichische Beiträge zu Meteorologie und Geophysik, No. 28, Zentralanstalt für Meteorologie und Geodynamik, Vienna, 304 pp

Auer I and 31 others (2007) HISTALP-historical instrumental climatological surface time series of the Greater Alpine Region. Intern. J. Climatol., 27, 17-46

Bai Z, Ding L and Yuz T (1985) Preliminary results of the investigation on the relations among the radiation and heat balance, glaciers and climate in the headwater of Urumqi river, Tianshan. Annual Report of Work at Tianshan Glaciological Station, 2, 199-241

Ballantyne CK (1989) The Loch Lomond readvance on the Island of Skye, Scotland: glacier reconstruction and palaeoclimatic implications. J. Quat. Sci., 4, 95-108

Barrueto A (2009) Accumulation on the Brewster Glacier in New Zealand. (MSc thesis, Department of Geography University Zurich), $88 \mathrm{pp}$

Benson CS (1962) Stratigraphic studies in the snow and firn of the Greenland ice sheet. SIPRE Res. Rep. 70, 93pp

Bessemoulin J (1989) Atlas Climatique de la France. Direction de la Météorologie, Ministrère des Transports, Paris

Böhm R and Hiebl J (2011/12) Lange instrumentelle Klimazeitreihen für den Vernagtferner. Z. Gletscherkd. Glazialgeol., 45/46, 203-218

Bradley RS (1975): Equilibrium-line altitudes, mass balance, and July freezing level heights in the Canadian High Arctic. J. Glaciol., 14, 267-274

Braithwaite RJ (2008) Temperature and precipitation climate at the equilibrium-line altitude of glaciers expressed by the degreeday factor for melting snow. J. Glaciol., 54(186), 437-444

Braithwaite RJ and Olesen OB (1984) Ice ablation in West Greenland in relation to air temperature and global radiation. Z. Gletscherkd. Glazialgeol., 20, 155-168

Braithwaite RJ, Raper SCB and Chutko K (2006) Accumulation at the equilibrium-line altitude of glaciers inferred from a degree-day 
model and tested against field observations. Ann. Glaciol., 43, 321-334

Brutsaert W (2005) Hydrology-an Introduction. Cambridge U. P., Cambridge, 605 pp

Budyko MI (ed.) (1963) Atlas teplovogo balansa zemnogo shara (Atlas of heat balance of the Earth). Akademiya Nauk, SSSR, Moscow, 69PP

Buffoni L and Chlistovsky F (1992) Precipitatzioni giornaliere rilevate all'Osservatorio Astronomico di Brera in Milano dal 1835 al 1990. edi-ermes, Milano, 148pp

Buffoni L, Magureri M and Nanni T (1999) Precipitation in Italy from 1833 to 1996. Theory Appl. Climatol., 63, 33-40

Calanca P-L and Heuberger R (1990) Energy balance. In Ohmura A, Lang $\mathrm{H}$, Blumer $\mathrm{F}$ and Grebner D, eds. Glacial climate research in the Tianshan, Zürcher Geographische Schriften, Heft 38, ETH Zürich, Zürich, 60-70

Cogley JG, Adams WR, Ecclestone MA, Jung-Rothenhäusler F and Ommanney CSL (1995) Mass Balance of Axel Heiberg Island Glaciers 1960-1991: A Reassessment and Discussion, NHRI Science Report No. 6, National Hydrological Research Institute, Saskatoon, $168 \mathrm{pp}$

Cogley JG and 10 others (2011) Glossary of Glacier Mass Balance and Related Terms. IHP-VII Technical Documents in Hydrology No.86, IACS Contribution No. 2, UNESCO-IHP, Paris, $114 \mathrm{pp}$

Dee DP and 35 others (2011) The ERA-interim reanalysis: configuration and performance of the data assimilation system. $Q$. J. $R$. Meteorol. Soc., 137, 553-597, doi: 10.1002/qj.828

de Leeuw J, Methven J and Blackburn M (2015) Evaluation of ERA-interim reanalysis precipitation products using England and Wales observations. Q. J. R. Meteorol. Soc., 141(688), 798-806

Dyurgerov M (2002) Glacier mass balance and regime: data of measurements and analysis. Occasional Paper 55, Institute of Arctic and Alpine Research, Univ. Colorado, Boulder, 88 pp

Escher-Vetter H (1980) Der Strahlungshaushalt des Vernachtferners als Basis der Energiehaushalt zur Bestimmung der Schmelzwasserproduktion eines Alpengletschers. Münchener UniversitätsSkripten, Fachbereich Physik, Wissenschaftliche Mitteilung, Nr.39, Meteorologisches Institut, Universität München, 115 pp

Escher-Vetter H (1985) Energy balance calculations from five years' meteorological records at Vernagtferner, Oetstal Alps. Z. Gletscherkd. Glazialgeol., 21, 397-402

Escher-Vetter H, Oerter H, Reinwarth O, Braun LN and Weber M (2012) Hydrological and meteorological records from the Vernagtferner Basin - Vernagtbach station, for the years 1970 to 2001. doi: 10.1594/PANGAEA.775113

Fliri F (1975) Das klima der Alpen im Raum von Tirol. Monographien zur Landeskunde Tirols. Folge 1, Wagner, Innsbruck, $454 \mathrm{pp}$

Forbes JD (1853) Norway and its glaciers visited in 1851. Adam and Charles Black, Edinburgh, 205-216

Forel F-A, Muret E and Mercanton P-L (1909) Débit du torrent glaciaire. Les variations périodiques des glaciers des Alpes suisses, 29th Report (1908 bis 1909), Jahrbuch des Schweizer Alpenclub, 44the year, 286-312

Fujii Y, Nakawo M and Shrestha ML (1976) Mass balance studies of the glaciers in Hidden Valley, Mukut Himal. Seppyo, 38, Special Issue, 17-21

Funk M (1985) Räumliche Verteilung der Massenbilanz auf dem Rhonegletscher und ihre Beziehung zu Klimaelementen. Zürcher Geogr. Schr. 24, 183pp

Funk M and Aellen M (Unpublished note, 1986) Bericht No.5026 über die Hydrologie und Glaziologie des Griesseegebietes im Jahr 1984/85 mit Uerbersicht der Hauptergebnisse seit 1967162 im Auftrag der Kraftweke Aegina AG, Ulrichen und Zürich, Juli 1986

Gerlitz L and 5 others (2016) A statistically based seasonal precipitation forecast model with automatic predictor selection and its application to central and south Asia. Hydrol. Earth Syst. Sci., 20, 4605-4623
Haeberli W (comp.) (1985) Fluctuations of glaciers 1975-1980, Vol. IV. International Association of Hydrological Sciences/UNESCO: Paris

Haeberli W and Herren E (ed.) (1991) Glacier mass balance bulletin, No. 1 (1988-1989), World Glacier Monitoring Service, Zurich

Haeberli W and Müller P (comp.) (1988) Fluctuations of glaciers 1980-1985, Vol. V. International Association of Hydrological Sciences/UNEP/UNESCO, Paris

Haeberli W, Herren E and Hoelzle M (ed.) (1993) Glacier mass balance bulletin, No. 2 (1990-1991), World Glacier Monitoring Service, Zurich

Haeberli W, Hoelzle M and Bösch M (ed.) (1994) Glacier mass balance bulletin, No. 3(1992-1993), World Glacier Monitoring Service, Zurich

Haeberli W, Hoelzle M and Suter S (ed.) (1996) Glacier mass balance bulletin, No. 4(1994-1995), World Glacier Monitoring Service, Zurich

Haeberli W, Hoelzle M and Frauenfelder R (ed.) (1999) Glacier mass balance bulletin, No. 5(1996-1997), World Glacier Monitoring Service, Zurich

Haeberli W, Frauenfelder R and Hoelzle M (ed.) (2001) Glacier mass balance bulletin, No. 6 (1998-1999), World Glacier Monitoring Service, Zurich

Haeberli W, Frauenfelder R, Hoelzle M and Zemp M (ed.) (2003) Glacier mass balance bulletin, No. 7 (2000-2001), World Glacier Monitoring Service, Zurich

Haeberli W, Noetzli J, Zemp M, Baumann S, Frauenfelder R and Hoelzle M (ed.) (2005) Glacier mass balance bulletin, No. 8 (2002-2003), World Glacier Monitoring Service, Zurich

Haeberli W, Hoelzle M and Zemp M (ed.) (2007) Glacier mass balance bulletin, No. 9 (2004-2005), World Glacier Monitoring Service, Zurich

Hann J (1883) Handbuch der Klimatologie. Engelhorn, Stuttgart, 764 pp Hansen BU and 9 others (2008) Present-day climate at Zackenberg. Adv. Ecol. Res., 40, 111-149

Hantke R (1978) Eiszeitalter, Vol. 1, Ott Verlag, Thun, 468 pp

Hattersley-Smith G and Serson H (1970) Mass balance of the Ward Hunt ice rise and Ice Shelf: 10 year record. J. Glaciol., 9 (56), 247-252

Havens JM (1964) Meteorology and heat balance on the accumulation area, McGill Ice Cap, Canadian Arctic Archipelago -summer 1960 (Upper Ice Station 1: $79^{\circ} 41$ N 90²7W, $1530 \mathrm{~m}$ ). Axel Heiberg Isl. Res. Rep., Meteorol. 2., McGill Univ., Montreal, 87pp

Higuchi K (1977) Effect of nocturnal precipitation on the mass balance of the Rikha Samba Glacier, Hidden Valley, Nepal. Seppyo 39, Special Issue, 43-49

Higuchi K, Ageta Y, Yasunari T and Inoue J (1982) Characteristics of precipitation during the monsoon season in high-mountain areas of the Nepal Himalaya. In Glen JW ed. Hydrological aspect of alpine and high-mountain areas, IAHS Publ., Wallingford, 138, 21-30

Hoinkes H (1955) Ueber Messungen der Ablation und des Waermeumsatzes auf Alpengletschern mit Bemerkungen ueber die Ursachen des Gletscherschwundes in den Alpen. IAHS Publ. No. 39, 442-448

Hoinkes H (1970) Methoden und Möglichkeiten von Massenhaushaltsstudien auf Gletschern. Z. Gletscherkd. Glazialgeol., 6(1-2), 38-90

Hoinkes $\mathrm{H}$ and Lang $\mathrm{H}$ (1962) Winterschneedecke und Gebietsniederschlag 1957/58 und 1958/59 im Bereich des Hintereis- und Kesselwandferners (Ötztaler Alpen). Arch. Meteorol. Geophgs. Bioklimatol. Ser. 8, 11(4), 424-446

Hoinkes $\mathrm{H}$ and Untersteiner N (1952) Wärmeumsatz und ablation auf Alpengletschern. Geogr. Ann., 34, 99-158

Holmgren B (1971) Climate and energy exchange on a sub-polar ice cap in summer. Arctic Institute of North America Devon Island Expedition 1961-1963. Part E. Radiation climate. Uppsala Universitet. Meteorologiska Institutionen. (Medde- lande 111.)

Holmlund P, Jansson P and Pettersson R (2005) A re-analysis of the 58 year mass balance record of Storglaciären, Sweden. Ann. Glaciol., 42, 389-394 
Huss M, Bauder A, Funk M and Hock R (2008) Determination of the seasonal mass balance of four Alpine glaciers since 1865. J. Geophys. Res., 113, F01015, doi: 10.1029/2007JF000803

IAHS/UNEP/UNESCO (1993) Fluctuations of glaciers 1985-1990, Vol. VI. World Glacier Monitoring Service, Zurich, Switzerland, $322 \mathrm{pp}$

IAHS/UNESCO (1998) Fluctuation of glaciers 1990-1995, Vol. VII, World Glacier Monitoring Service, Zurich, Switzerland, $296 \mathrm{pp}$

Isotta $\mathrm{F}$ and 17 others (2014) The climate of daily precipitation in the Alps: development and analysis of a high-resolution grid dataset from pan-Alpine rain-gauge data. Int. J. Climatol., 34, 1657-1675

IUGG(CCS)/UNEP/UNESCO (2005) Fluctuations of glaciers 19952000, Vol. VIII. World Glacier Monitoring Service, Zurich, Switzerland, $288 \mathrm{pp}$

Japanese Antarctic Research Expedition (1985) Science in Antarctica, Vol. 9, Data compilation Tokyo, National Institute of Polar Research, Tokyo

Jin $\mathrm{H}$ and Wang J (2002) Role of permafrost and water retaining forests in the mountain runoff formation. Chapter. 6. In Kang E, Chen C and Dong Z, eds. Glacier water resources and mountain runoff in the arid area of Northwest China, Science Press, Beijing, 125-155

Kang E, Shi Y, Ohmura A and Lang H (1996) Runoff formation and discharge modelling of a glacierized basin in the Tienshan Mountains. In Jones JAA and others eds. Regional hydrological response to climate change, Kluwer, Dortrecht, 241-257

Kasser P (comp.) (1967) Fluctuation of glaciers 1959-1965. Paris, International Association of Hydrological Sciences/UNESCO

Kjøllmoen B (ed.) (2011) Glaciological investigations in Norway in 2010. Norwegian Water Resources and Energy Directorate, Oslo, $89 \mathrm{pp}$

Koerner RM (1966) Accumulation on the Devon Island ice cap, northwest territories, Canada. J. Glaciol., 6(45), 383-392

Koerner RM (1970) The mass balance of the Devon Island Ice Cap, northwest territories, 1961-66. J. Glaciol., 9, 325-336

Koerner RM (1979) Accumulation, ablation; and oxygen isotope variations on the Queen Elizabeth Islands ice caps, Canada. J. Glaciol., 22(86), 25-41

Kotlyakov VM and Krenke AN (1982) Investigation of the hydrological conditions of alpine regions by glaciological methods. IAHS Publ., 138, 31-42

Kraus $\mathrm{P}$ (1983) Glacier inventory of southeast Ellesmere Island, N.W. T., Canada and its application for estimating the annual run-off. Zürcher Geogr. Schr. 9

Krenke AN and Khodakov VG (1997) On the correlation between glacier melting and air temperature. In Kotlyakov VM, ed. 34 selected papers on main ideas of the Soviet glaciology 1940s1980s. Moscow, Inst. Geogr., RAS, 191-207. (originally published in 1966 under the same title from Materialy Glyatsiologicheskikh Issledovanii Khronika Obsuzhdeniya, Moscow, No.12, 153-164)

Kuhn M (1980) Die Reaktion der Schneegrenze auf Klimaschwankungen. Z. Gletscherk. Glazialgeol., 16(2), 241254

Kuhn M (1981) Climate and glaciers. International Association of Hydrological Sciences Publication 131 (Symposium at Canberra 1979 Sea Level, Ice, and Climatic Change), 3-20

Kuhn M (1989) The response of the equilibrium line altitude to climate fluctuations; theory and observations. In Oerlemans J, ed. Glacier fluctuations and climatic change. Kluwer, Dortrecht, 407-417

Kulkarni AV (1992) Mass balance of Himalayan glaciers using AAR and ELA methods. J. Glaciol., 38(128), 101-104

LaChapelle E (1959) Annual mass and energy exchange on the Blue Glacier. J. Geophys. Res., 64, 443-449

Lang H (1968) Relations between glacier runoff and meteorological factors observed on and outside the glacier. IAHS Publ., 79, 429-439

Liestøl O (1967) Storbreen glacier in Jotunheimen, Norsk Polarinstitutt Skrifter, Oslo, Norway, No. 141, 63 pp
Lister $\mathrm{H}$ (1962) Heat and mass balance at the surface of Ward Hunt Ice Shelf, 1960. Research Paper No. 19, Arctic Institute of North America, Montreal, 54pp

Liu S, Sun W, Shen Y and Li G (2003) Glacier changes since the little ice age maximum in the western Qilian Mountains, northwest China. J. Glaciol., 49(164), 117-124

Loewe $\mathrm{F}$ (1971) Considerations on the origin of the quaternary ice sheet of North America. Arct. Alp. Res., 3, 331-344

Lundqvist M ed. (1953) Atlas över Sverige. Svenska Sällskapet för antropologi och geografi, Stockholm, $150 \mathrm{pp}$

Mani A (1980) Handbook of solar radiation data for India. etc., Allied Publishers, New Delhi

Marcus MG (1964) Climate-glacier studies in the Juneau Ice Field region. University of Chicago Press, Alaska. Chicago. (Department of Geography Research Paper 88.)

Marshunova MS and Chernigovskiy NT (1971) Radiatszonnyy rezhim zarubezhnoy Arktiki (radiation regime of the foreign Arctic). Gidrometeorologicheskoye Izdatel'stvo, Leningrad, 181pp

Marty C (2000) Surface radiation, cloud forcing and greenhouse effect in the Alps. (PhD thesis, E.T.H. Zurich), $141 \mathrm{pp}$

Maurer J (1914) Die jüngste grosse Rückzugsphase der schweizer Gletscher im Lichte der Klimaschwankung. Dr A. Petermanns Mitteilungen aus Justus Perthes' Geographischer Anstalt, 10-12

Meier MF, Tangborn WV, Mayo LR and Post A (1971) Combined ice and water balances of Gulkana and Wolverine glaciers, Alaska and South Cascade Glacier, Washington, 1965 and 1966 hydrologic years. U.S. Geol. Surv. Prof. Pap.725-A

Mernild S and 5 others (2013) Identification of snoa ablation rate, ELA, AAR and net mass balance using transient snow line variations on two Arctic glaciers. J. Glaciol., 59(216), 649-659

Mokievsky-Zubok O and Stanley AD (1976a) Canadian glaciers in the International Hydrological Decade Program, 1965-1974. No. 1. Sentinel Glacier, British Columbia-summary of measurements. Ottawa, Inland Waters Directorate. Water Resources Branch. (Scientific Series 68.)

Mokievsky-Zubok O and Stanley AD (1976b) Canadian glaciers in the International Hydrological Decade Program, 1965-1974. No. 2. Place Glacier, British Columbia-summary of measurements. Ottawa, Inland Waters Directorate. Water Resources Branch. (Scientific Series 69.)

Mokievsky-Zubok O, Ommanney CSL and Power J (1985) NHRI glacier mass balance 1964-1984 (Cordillera and Arctic). Environment Canada. National Hydrology Research Institute, Ottawa. Surface Water Division. Glacier Section

Moser $\mathrm{H}$, Escher-Vetter $\mathrm{H}$, Oerter $\mathrm{H}$, Reinwarth $\mathrm{O}$ and Zunke D (1986) Abfluss in und von Gletschern. Munich, Gesellschaft für Strahlen- und Umweltforschung. GSF-Bericht 4I/86

Müller F (comp.) (1977) Fluctuations of glaciers 1970-1975, Vol. III, International Association of Hydrological Sciences/UNESCO, Paris

Müller-Lemans H, Kappenberger G and Steinegger U (1993) Methodische Aspekte der Niederschlagsbestimmung im Alpenraum. In Grebner D, ed. Aktuelle Aspekte in der Hydrologie. Festschrift zum 60. Geburtstag von Herbert Lang, Zürich. Geogr. Schr., Haft 53, 96-107

Müller-Lemans $\mathrm{H}$, Aellen M, Braun LN, Kappenberger $\mathrm{G}$ and Steinegger U (1997) Niederschlagsverteilung im Tödigebiet: Messungen und Überprüfung. In Moser U, ed. Niederschlag und Wasserhaushalt im Hochgebirge der Glarner Alpen. Beiträge zur Hydrologie der Schweiz, Nr. 36, Schweiz. Gesell. Hydrol. und Limnologie, Birmensdorf, 7-43

Nesje A and Dahl SO (2000) Glaciers and environmental change, key issues in environmental change. Arnold, London, 203 pp

Ohmura A (1977) Radiation and heat balance on the surface of north water. In Müller F, ed. North water project. Progress Report 1 October 1975 to 30 September 1976. Zürich, ETH; Montreal, McGill University, 41-54

Ohmura A (1981) Climate and energy balance on Arctic tundra, Axel Heiberg Island, Canadian Arctic Archipelago, spring and summer 1969, 1970 and 1972. Zürcher Geogr. Schr. 3 
Ohmura A (1982) Climate and energy balance on the Arctic tundra. J. Climatol., 2, 65-84

Ohmura A (ed.) (1991) ETH Greenland Expedition, Progress Report No. 1: April 1990 to February 1991. Department of Geography, Swiss Federal Institute of Technology, Zurich, 108 pp

Ohmura A (ed.) (1992) ETH Greenland Expedition, Progress Report No. 2: April 1991 to October 1992. Department of Geography, Swiss Federal Institute of Technology, Zurich, 94 pp

Ohmura A (2001) Physical basis for the temperature/melt-index method. J. Appl. Meteor., 40, 753-761

Ohmura A and Müller F (1978) Aspects of mesoscale distribution of air temperature and precipitation over high Arctic tundra. Polar Geogr., 2, 270-284

Ohmura A, Lang H, Blumer F and Grebner D (eds.) (1990) Glacial climate research in the Tienshans: Research Report on Project Glacier No.1, 1985-1987. Zürcher Klima Schriften No. 38, Inst. Climate Res., Swiss Federal Institute of Technology (E.T. H.), Zurich, $181 \mathrm{pp}$

Ohmura A, Kasser P and Funk M (1992) Climate at the equilibrium line of glaciers. J. Glaciol., 38(130), 397-411

Ohmura A and 6 others (1994) Energy balance for the Greenland ice sheet by observation and model computation. In Jones HG, Davies TD, Ohmura A and Morris EM, eds. Snow and Ice covers; interactions with the atmosphere and ecosystems, IAHS Publ., Wallingford, No. 223, 85-94

Ohmura A, Calanca P, Wild M and Anklin M (1999) Precipitation, accumulation and mass balance of the Greenland ice sheet. $Z$. Gletscherk. Glazialgeol., 35, 1-20

Ohmura A, Bauder A, Müller H and Kappenberger G (2007) Longterm change of mass balance and the role of radiation. Ann. Glaciol., 46, 367-374

Olesen OB (1986) Fourth year of glaciological field work at Tasersiaq and Qapiarfiup sermia, West Greenland. Grønl. Geol. Undersøgelse. Rapp. 130, 121-126

Østrem G and Liestøl O (eds.) (1964) Glasiologiske undersøkelser i Norge 1963. Norsk Polarinstitutt Meddel. No. 90, 59pp

Palz W, Kasten F and Golchert HJ (eds.) (1979) European solar radiation atlas, Vol. 1. Global radiations on horizontal surfaces. W. Grösschen Verlag, Dortmund

Paterson WSB (1969) The physics of glaciers. Pergamon Press, Oxford and others, $250 \mathrm{pp}$

Pellicciotti $F$ and 5 others (2005) An enhanced temperature-index glacier melt model including the shortwave radiation balance: development and testing for Haut Glacier d'Arolla, Switzerland. J. Glaciol., 51(175), 573-587

Pelto M, Kavanaugh J and McNeil C (2013) Juneau icefield mass balance program 1946-2011. Earth Syst. Sci. Data, 5, 319330, doi: 10.5194/essd-5-319-2013

Priestley CHB (1959) Turbulent transfer in the lower atmosphere. University Chicago Press, Chicago, 130 pp

Rasmussen L and Wenger J (2009) Upper-air model of summer balance on Mt. Rainier, USA. J. Glaciol., 55(192), 619-624

Sagar RB (1964) Meteorological and glaciological observations on the Gilman Glacier, northern Ellesmere Island, 1961. Geogr. Bull., 22, 13-56

Scherhag R (1969) Klimatische Karten der Nordhemisphäre. Freie Universität Berlin, Institut für Meteorologie und Geophysik, Meteorologische Abhandlungen, 100(1)

Schüepp M, Bouët $M$, Bider M and Urfer C (1978) Regionale Klimabeschreibungen, 1. Teil Gesamtübersicht, Westschweiz, Wallis, Jura und Juranordfuss sowie Mittelland. Klimatologie der Schweiz, Bd. 2, Schweizrische Meteorologische Zentralanstalt, Zurich, $245 \mathrm{pp}$

Schwerdtfeger W (1984) Weather and climate of the Antarctic. Elsevier, Amsterdam (Developments in Atmospheric Science 15.), 261 pp

Schytt V (1955) Glaciological investigations in the Thule Ramp area. SIPRE Res. Rep. 28

Schytt V (1981) The net mass balance of Storglaciaren, Kebnekaise, Sweden related to the height of the equilibrium line and the height of the $500 \mathrm{mb}$ surface. Geogr. Ann., 630A 219-223

Service de la Météorologie, Québec (1978) Atlas Climatologique du Québec. Ministère des richesses naturelles, Direction Général des Eaux, $42 \mathrm{pp}$

Seversky IV (1997) On the procedure of evaluating average annual sums of solid precipitation on an equilibrium line of glaciers. In Kotlyakov VM, ed. 34 selected papers on main ideas of the Soviet glaciology 1940s-1980s. Inst. Geogr., RAS, Moscow, 347-354. (Originally published in 1978 under the same title from Contribution of Academy of Sciences, Kazakhstan Soviet Socialist Republic, Vol. 11, 43-50)

Shanklin JD (1981) Measurements of solar and terrestrial radiation at King Edward Point, South Georgia, 1973 81. British Antarctic Survey, Cambridge

Tangborn WV, Mayo LR, Scully DR and Krimmel RM (1977) Combined ice and water balances of Maclure Glacier, California, South Cascade Glacier, Washington, and Wolverine and Gulkanl glaciers, Alaska, 1967 Hydrological Year. U.S. Geol. Surv. Prof. Pap.725-B

Tanzer G (1986) Berechnung des Wärmehaushalts an der Gleichgewichtslinie des Hintereisferners. Diplomarbeit. University Innsbruck, Innsbruck, 103 pp

Taylor-Alt B (1975) The energy balance climate of Meighen Ice Cap, N.W.T., Vol. 1 and 2, Polar Continental Shelf Project, Energy, Mines and Resources Canada, Ottawa, 168 pp

Thomson LI, Zemp M, Copland L, Cogley JG and Ecclestone MA (2017) Comparison of geodetic and glaciological mass budgets for White Glacier, Axel Heiberg Island, Canada. J. Glaciol., 63 (237), 55-66. Doi: 10.1027/jog.2016.112

Timmis RJ (1986) Glacier changes in South Georgia and their relationship to climatic trends. (PhD thesis, University of East Anglia)

Vincent C (2002) Influence of climate change over the 20th century on four French glacier mass balances. J. Geophys. Res., 107 (D19), 4375 doi: 10.1029/2001JD000832

Wagner HP (1978) Strahlungshaushaltsuntersuchungen am Hintereisferner/Ötztal wärend der Hauptablationsperiode 1971. (PhD thesis, University Innsbruck) $188 \mathrm{pp}$

Wagner HP (1979) Strahlungshaushaltsuntersuchungen an einem Ostalpengletscher während der Hauptablationsperiode, Teil 1: Kurzwellige Strahlung. Arch. Meteorol. Geophys. Bioklim. Ser. $B, 27,297-324$

Wagner HP (1980) Strahlungshaushaltsuntersuchung an einem Ostalpengletscher während der Hauptablationsperiode, Teil 2: Langwellige Strahlung und Strahlungsbilanz. Arch. Met. Geophys. Biokl., Ser. B, 28, 41-62

Wagnon P, Ribstein P, Francou B and Pouyaud B (1999) Annual cycle of energy balance of Zongo glacier, Cordillera Real, Bolivia. J. Geophys. Res., 104(D4), 3907-3923

Wang $N$ and 5 others (2009) Tracing the major source area of the mountainous runoff generation of the Heihe River in northwest China using stable isotope technique. Chin. Sci. Bull., 54, 2751-2757 doi: 10.1007/s11434-009-0505-8

Wang N, He J, Pu J, Jiang X and Jing Z (2010) Variations in equilibrium line altitude of the Qiyi Glacier, Qilian Mountains, over the past 50 years. Chin. Sci. Bull., 55, 3810-3817

Weber M (1997) Aspekte zur Extrapolation von Tagesmittelwerten von Temperatur und täglichen Niederschlagssummen an hochgelegenen Gebirgsstationen aus Klimadaten des örtlichen Klimamessnetzes in Nepal. Interner Bericht, Kommission für Glaziologie der Bayerischen Akademie der Wissenschaften, Munich, 34pp

Weidick A and Thomsen HH (1986) A decade of glacier investigations for utilisation of Greenland hydropower. Grønl. Geol. Undersøgelse. Rapp. 128, 157-169

Weiss J (1984) Massenhaushalt eines Gletscher-Fragen an glaziologische Untersuchungen und die Resultate der Messreihe am White Glacier in der kanadis- chen Arktis. Diplomarbeit, Eidgenössische Technische Hochschule, Zürich 
WGMS (2008) Fluctuations of Glaciers 1985-1990. Vol. IX., Haeberli W, Zemp M, Kääb A, Paul F, and Hoelzle M (eds.), ICSU(FAGS)/ IUGG(IACS)/UNEP/UNESCO/WMO, World Glacier Monitoring Service, zurich Switzerland, $266 \mathrm{pp}$

WGMS (2009) Glacier Mass Balance Bulletin, No. 10 (2006-2007). Haeberli W, Gärtner-Roer I, Hoelzle M, Paul F, and Zemp M (eds.), ICSU(WDS)/IUGG(CCS)/UNEP/UNESCO/WMO, World Glacier Monitoring Service, Zurich, Switzerland, $96 \mathrm{pp}$

WGMS (2011) Glacier Mass Balance Bulletin, Bulletin No. 11 (2008-2009). Zemp M, Nussbaumer SU, Gärtner-Roer I, Hoelzle M, Paul F, Haeberli W (eds.), ICSU(WDS)/IUGG(IACS)/ UNEP/UNESCO/WMO, World Glacier Monitoring Service, Zurich, Switzerland, 102 pp

WGMS (2012) Fluctuations of Glaciers 2000-2005. Vol. X. Zemp M, Frey H, Gärtner-Roer I, Nussbaumer SU, Hoelzle M, Paul F, and Haeberli W, (eds.), ICSU(WDS)/IUGG(IACS)/UNEP/UNESCO/ WMO, World Glacier Monitoring Service, Zurich, Switzerland

WGMS (2013) Glacier Mass Balance Bulletin, No. 12 (2010-2011). Zemp, M, Nussbaumer SU, Naegeli K, Gärtner-Roer I, Paul F, Hoelzle M, and Haeberli W (eds.), ICSU(WDS)/ IUGG(CCS)/ UNEP/UNESCO/WMO, World Glacier Monitoring Service, Zurich, Switzerland, $106 \mathrm{pp}$

WGMS (2015) Global Glacier Change Bulletin, No.1 (2012-2013). Zemp M, Gärtner-Roer I, Nussbaumer SU, Hüsler F, Machguth $\mathrm{H}$, Mölg N, Paul F, and Hoelzle M (eds.), ICSU(WDS)/IUGG (IACS)/UNEP/UNESCO/WMO, World Glacier Monitoring Service, Zurich, Switzerland
Wild $\mathrm{M}$ and 5 others (1998) The disposition of radiative energy in global climate system: GCM-calculated versus observational estimates. Clim. Dyn., 14, 853-869

Wild M and 7 others (2017) The Global Energy Balance Archive (GEBA): a database for worldwide measured surface energy fluxes. Earth Syst. Sci. Data, 9, 1-12

Xie Z (1984) Studies of ice formation and ice fabric on the Law Dome, Antarctica. J. Glaciol. Cryopedol., 6(1), 1-23

Yasunari T and Inoue J (1978) Characteristics of monsoon precipitation around peaks and ridges in Shorong and Khumbu Himal. Seppyo, 40, Special Issue, 26-32

Young GJ and Stanley AD (1976a) Canadian glaciers in the International Hydrological Decade Program, 1965-1974. No. 3. Ram River Glacier, Alberta, summary of measurements. Scientific Series 70, Inland Waters Directorate. Water Resources Branch, Ottawa

Young GJ and Stanley AD (1976b) Canadian glaciers in the International Hydrological Decade Program, 1965-1974. No. 4. Peyto Glacier, Alberta-summary of measurements. Scientific Series 71, Inland Waters Directorate. Water Resources Branch, Ottawa

Zemp $M$ and 5 others (comp.) (2011) Glacier Mass Balance Bulletin, Bulletin No. 11 (2008-2009). ICSU(WDS)/IUGG(IACS)/UNEP/ UNESCOMMO. World Glacier Monitoring Service, Zurich

Zemp M and 6 others (comp.) (2012) Fluctuations of Glaciers 20002005. Vol. X. Paris, ICSU(WDS)/IUGG(IACS)/UNEP/UNESCO/ WMO

MS received 1 March 2018 and accepted in revised form 17 April 2018; first published online 21 May 2018 